\title{
Many-Body Physics in the NISQ Era: Quantum Programming a Discrete Time Crystal
}

\author{
Matteo Ippoliti®, ${ }^{1,{ }^{*}}$ Kostyantyn Kechedzhi, ${ }^{2}$ Roderich Moessner, ${ }^{3}$ S.L. Sondhi, ${ }^{4}$ and Vedika Khemani ${ }^{1}$ \\ ${ }^{1}$ Department of Physics, Stanford University, Stanford, California 94305, USA \\ ${ }^{2}$ Google Research, Venice, California 90291, USA \\ ${ }^{3}$ Max-Planck-Institut für Physik komplexer Systeme, Dresden 01187, Germany \\ ${ }^{4}$ Department of Physics, Princeton University, Princeton, New Jersey 08540, USA
}

(Received 24 August 2020; accepted 9 August 2021; published 20 September 2021; corrected 29 July 2022)

Recent progress in the realm of noisy intermediate-scale quantum (NISQ) devices [J. Preskill, Quantum 2, 79 (2018)] represents an exciting opportunity for many-body physics by introducing new laboratory platforms with unprecedented control and measurement capabilities. We explore the implications of NISQ platforms for many-body physics in a practical sense: we ask which physical phenomena, in the domain of quantum statistical mechanics, they may realize more readily than traditional experimental platforms. While a universal quantum computer can simulate any system, the eponymous noise inherent to NISQ devices practically favors certain simulation tasks over others in the near term. As a particularly wellsuited target, we identify discrete time crystals (DTCs), novel nonequilibrium states of matter that break time translation symmetry. These can only be realized in the intrinsically out-of-equilibrium setting of periodically driven quantum systems stabilized by disorder-induced many-body localization. While promising precursors of the DTC have been observed across a variety of experimental platforms - ranging from trapped ions to nitrogen-vacancy centers to NMR crystals - none have all the necessary ingredients for realizing a fully fledged incarnation of this phase, and for detecting its signature long-range spatiotemporal order. We show that a new generation of quantum simulators can be programmed to realize the DTC phase and to experimentally detect its dynamical properties, a task requiring extensive capabilities for programmability, initialization, and readout. Specifically, the architecture of Google's Sycamore processor is a remarkably close match for the task at hand. We also discuss the effects of environmental decoherence, and how they can be distinguished from 'internal' decoherence coming from closed-system thermalization dynamics. Already with existing technology and noise levels, we find that DTC spatiotemporal order would be observable over hundreds of periods, with parametric improvements to come as the hardware advances.

DOI: 10.1103/PRXQuantum.2.030346

\section{INTRODUCTION}

The quest to build a universal quantum computer has fueled sustained progress towards the development of "designer" many-body quantum systems across a variety of platforms ranging from trapped ions to superconducting qubits $[1,2]$. While the ultimate goal of a fault-tolerant quantum computer is still far into the future, the possibility of harnessing the computational power of the quantum world with noisy intermediate-scale quantum

\footnotetext{
*ippoliti@stanford.edu

Published by the American Physical Society under the terms of the Creative Commons Attribution 4.0 International license. Further distribution of this work must maintain attribution to the author(s) and the published article's title, journal citation, and DOI.
}

(NISQ) [3] devices is already a reality. A notable milestone in this context was the recent announcement of "quantum supremacy" (more accurately, "quantum computational supremacy" [4]) in Google's Sycamore device, a solid-state, Josephson-junction-based platform with 53 qubits [5]. While the computational task chosen for this purpose - simulating the output of random quantum circuits - may seem rather abstract and not useful in and of itself (though it does have at least one application [6]), a very active search for high-impact applications of NISQ devices is underway. In this vein, two recent works discussed how to implement highly structured circuits for quantum chemistry simulations [7] and combinatorial optimization problems [8] on Sycamore.

Now, a quantum computer is also necessarily a highly controllable many-body system [9], and so these advances are also extremely tantalizing to many-body physicists 
looking to push the frontiers of their own discipline. Indeed, Google's announcement, signifying a major breakthrough in computational science, also heralded the advent of a new laboratory system with Hilbert spaces of significant size, which can potentially be used to host and discover new many-body physics.

This paper is motivated, broadly, by asking what the NISQ era of tunable, programmable quantum systems portends for many-body physics; and, narrowly, by asking what interesting physics could be realized immediately with Google's device. Which physical phenomena in the realm of quantum statistical mechanics can these devices realize, that have not yet been (as) crisply demonstrated in any other experimental setting? As with the random circuit problem, a first demonstration should perhaps explore a landscape where some landmarks are already known and can be used to guide the search while leaving room for discovery.

Two conceptual challenges immediately present themselves to the many-body physicist. (i) The natural time evolutions implemented on digital gate-based programmable simulators (such as Sycamore) are quantum circuits rather than Hamiltonians. This is quite far from the typical setting in which condensed matter theory operates, which concerns the low-energy, long-wavelength emergent properties of equilibrium many-body systems. This is also distinct from regimes probed by analog simulators, such as cold-atom platforms, which generally target specific model Hamiltonians [10,11]. And (ii) the tradeoffs between unitary control and platform size inevitably build some variation in individual circuit elements, which presents an additional challenge for simulating finely tuned model systems. We emphasize here that we are not viewing these platforms as universal computational devices that can simulate any desired unitary evolution $[1,12,13]$ or allow computational investigation of the properties of particular Hamiltonians and quantum states [14-16]. Instead, due to near-term limitations in size and coherence time, we are interested in identifying physical phenomena that these platforms can immediately and naturally realize, as opposed to physics they could realize universally and asymptotically.

A parallel set of developments in quantum statistical mechanics furnishes a domain where these specific challenges turn into strengths: the study of nonequilibrium dynamics, and specifically the assignation of robust phase structure to many-body systems out of equilibrium. Remarkably, even without the conceptual framework of equilibrium thermodynamics, a possibility to identify phases and phase transitions remains [17-19]. This line of research has led to the discovery of new kinds of dynamical many-body phenomena that may otherwise be forbidden by the strictures of equilibrium thermodynamics, with the discrete time crystal (DTC) phase being the first and most paradigmatic example of this phenomenon [19-23].
Combining these insights leads us to focus on dynamical phases in disordered, out-of-equilibrium quantum matter - specifically, many-body localized (MBL) periodically driven (or Floquet) phases - as natural candidates for the NISQ-era scientific program outlined above. Indeed, the quantum circuit structure that is Sycamore's modus operandi lends itself naturally to implement various Floquet drive protocols. Further, for these applications, randomness in circuit elements is not only tolerated, but is in fact necessary to stabilize the system against heating, and thus for observing interesting phenomena. For these reasons, in this work we propose precisely such a 'physicsforward" use of the Sycamore device and its relatives: to realize a MBL Floquet DTC, a nonequilibrium manybody phase of matter that displays an entirely new form of spatiotemporal order [19-21]. One striking feature of the DTC phase is that it spontaneously breaks the discrete time translation symmetry of the drive and exhibits period doubling, a dynamical phenomenon with a long and rich history $[24,25]$ that has recently seen a resurgence in interest, with proposals spanning a wide range of classical and quantum systems [26-32].

Our choice has several desirable aspects: (i) the DTC is a genuine collective many-body phenomenon, and represents the best known example of a new paradigm in quantum statistical mechanics, that of an out-of-equilibrium phase of matter; (ii) it is of clear fundamental and conceptual importance, given its distinctive pattern of spatiotemporal order; and (iii) despite promising precursors [33-36], a bona fide realization of this phase (or any manybody out-of-equilibrium phase, for that matter) has proved elusive for differing reasons in each of the existing experimental platforms in which it has been explored. Indeed, as we explain below, there are fundamental definitional aspects of the physics of this phase, specifically its central attributes of spatiotemporal order and robustness to choice of initial state, that have not yet been observed [22]. Not only have these not been observed, detailed theoretical analysis has shown that these defining features are fundamentally absent in the state-of-the-art experiments probing the DTC [22]. Thus, this proposal is not about repeating previous experiments with incremental extensions to the scope of their observations; rather, it is about realizing and demonstrating the first genuine instance of this phase.

There is much reason to be optimistic. The prior impressive experimental studies on DTCs have enabled a detailed understanding of the remaining obstacles to the realization of this phase, so that this goal appears eminently achievable in the near term. The resulting checklist contains several requirements that are hard to simultaneously satisfy in the previous setups. But these are sufficiently well defined to be individually addressed and simultaneously realized on the Sycamore device. Indeed, as we show in this work, the existing capabilities, architecture, and gate 
set in Sycamore satisfy all the desiderata, and the platform seems almost tailor made for this application.

We flesh out our proposal as follows. Section II A contains a telegraphic account of the basics of DTCs to orient the following discussion. Section II B presents a detailed account of the insights from previous experiments, from which we distill a list of experimental desiderata in Sec. II C. Section III details how to meet these, and explains how to address the implementation of the required experimental protocol on a present-day quantum device, Google's Sycamore processor. We then provide evidence that the phenomenon we are looking for is indeed present for a range of experimentally achievable parameters (Sec. III B), and present an analysis of noise and other experimental imperfections to argue that its observation is possible despite present limitations of the NISQ platform (Sec. IV). We conclude by discussing our results and directions for future work in Sec. V.

\section{THE DISCRETE TIME CRYSTAL: THEORY AND EXPERIMENTS}

We begin by briefly recapitulating the physics of the DTC phase in Sec. II A, which defines the model and notation. This provides a minimal set of facts about the DTC needed to render this article self-contained; it may therefore be read diagonally by those with prior exposure to the field. Section II B discusses the state of the art in experimental efforts to engineer the DTC, followed in Sec. II C by the enumeration of an experimental checklist of ingredients for realizing and observing this phase. These have not been simultaneously achievable in any single platform thus far. We refer the reader interested in an in-depth account of these issues to a review on time crystals by some of the present authors [22].

\section{A. Theoretical definitions and models}

\section{The DTC and its import}

The canonical model of a (discrete) time crystal [19] is realized in a Floquet system with a time-periodic Hamiltonian, with discrete time translation symmetry (DTTS) $H(t)=H(t+T)$. A DTC spontaneously breaks the DTTS of the drive: observables in this phase show periodic dynamics with a period $m T$, with $\mathbb{Z} \ni m>1$, corresponding to a sharp subharmonic response in the frequency domain (for example, $m=2$ for period-doubled dynamics).

The search for a time crystal has roots dating back to age-old quests for perpetual motion machines, and this is a phase of matter that is provably disallowed by the strictures of equilibrium thermodynamics [22,37]. Hence, the intrinsically nonequilibrium setting of a periodically driven system is constitutive to realizing a time crystal.
Period doubling (or multiplexing) is ubiquitous in classical and quantum dynamical systems, in settings ranging from Faraday waves to parametric oscillators [24, $25,38]$. However, these examples arise in single- or fewbody systems, or in systems that are effectively few-body (in a mean-field sense) [22]. On the other hand, defining a time crystal as a nontrivial, many-body phase of matter requires us to consider macroscopic, strongly interacting quantum systems. This is, in fact, the only setting in which time translation symmetry breaking is unexpected from the viewpoint of equilibrium thermodynamics; oneor few-body systems, such as simple harmonic oscillators, routinely exhibit oscillations and revivals in their dynamics.

A pervasive challenge with periodically driven manybody systems is their tendency to absorb energy from the drive and thermalize to infinite temperature, maximizing entropy in the absence of conservation laws $[39,40]$. One robust mechanism for escaping this "heat death" is manybody localization (MBL), wherein the dynamics fails to establish local thermal equilibrium even at arbitrarily late times due to disorder [41-46]. In particular, the system is thus prevented from heating to a trivial state.

A most striking property of this many-body localized phase is that it can now support new forms of order, which can be defined despite the inapplicability of the usual and familiar framework of equilibrium thermodynamics. The assignation of robust phase structure without relying on ground states or equilibrium Gibbs states (or even time-independent Hamiltonians) is a fundamentally new paradigm in many-body physics, and the framework goes under the name of eigenstate order [18]. Most simply, many-body eigenstates of the system's Hamiltonian (or Floquet unitary) may individually display nontrivial order and correlations, even as averages over eigenstates, such as in a Gibbs state, show no order. For example, the eigenstates may come in pairs, related to each other in the same way as the symmetry-broken ground states of a standard Ising ferromagnet. Unlike the latter, however, the pairing of states will be present throughout the (quasi)energy spectrum, with measurable dynamical consequences starting from states at all energies (instead of merely low-temperature ones).

Eigenstate order of this type then underpins various nontrivial nonequilibrium phases, of both the symmetrybreaking and topological varieties. For a brief pedagogical introduction to nonequilibrium phase structure in Floquet systems, see Ref. [47].

In sum, the importance of the DTC is based on two pillars. First, it exhibits the spontaneous time translation symmetry breaking expressed in its name, thereby closing out a centuries old quest for time crystals and capturing the imagination of the general public. Second, since such time translational symmetry breaking and 
spatiotemporal order is absent from all equilibrium phases, it stands out as, arguably, the most distinctive and striking instance of a new paradigm in many-body physics: an eigenstate-order-based nonequilibrium phase of manybody matter.

\section{Model realizations}

We now turn to specific model realizations of this phase. A standard model of a Floquet DTC is an Ising model periodically "kicked" by a rotation about the $\hat{x}$ axis [19]. The dynamics probed at "stroboscopic" times, $t=n T, n \in \mathbb{Z}$, is captured by studying the properties of the "Floquet unitary," which is the time-evolution operator over one period,

$$
U_{F}=e^{-i g \sum_{i} X_{i}} e^{-i T\left(H_{z}+H_{\text {int }}\right)},
$$

where $T \equiv 1$ is the drive period, the $X_{i}\left(Z_{i}\right)$ denote spin$1 / 2$ Pauli $x(z)$ operators on site $i, H_{z}=\sum_{i, j} J_{i j} Z_{i} Z_{j}$ is a diagonal Hamiltonian with Ising symmetry $P=\prod_{i} X_{i}$, and $H_{\text {int }}$ represents additional generic interactions that may be present (examples include longitudinal fields $H_{\text {int }}=$ $\sum_{i} h_{i} Z_{i}$ or $X Y$ interactions $\left.H_{\mathrm{int}}=\sum_{i j} J_{i j}^{\perp}\left[X_{i} X_{j}+Y_{i} Y_{j}\right]\right)$. Localizing the system to prevent heating will require disorder in the couplings $J_{i j}$.

The model in Eq. (1) can potentially realize a discrete time-crystal phase in the regime $g=\frac{1}{2}(\pi-\epsilon)$, with $\epsilon$ sufficiently small. This represents an imperfect " $\pi$ pulse," i.e., a nearly $180^{\circ}$ rotation about the $x$ axis. To understand the properties of the phase, consider first the limit $\epsilon=H_{\text {int }}=$ 0 . In this case, it easy to see that starting with a product state in the $\hat{z}$ basis, one action of the unitary enacts a perfect $180^{\circ}$ rotation and flips all spins; these are then flipped back under a second action of $U(T)$, thereby showing period-doubled dynamics, $\left\langle Z_{i}(m T)\right\rangle=(-1)^{m}\left\langle Z_{i}(0)\right\rangle$.

While the $\epsilon=H_{\text {int }}=0$ limit is illustrative, defining the DTC as a phase of matter requires some degree of stability to the choice of parameters and interactions. Indeed, what is remarkable is that, under suitable conditions (requiring the presence of MBL), the dynamics can remain robustly locked at period doubling for infinitely long times in an extended region of parameter space, i.e., even for imperfect rotations $(\epsilon \neq 0)$ and in the presence of generic perturbing interactions $\left(H_{\text {int }} \neq 0\right)$ [19-21]. We emphasize that this stability is inexplicable using any kind of semiclassical intuition; without quantum ordering, one would expect a finite deviation in rotation angle $(\epsilon \neq 0)$ to accumulate over consecutive cycles, destroying the period doubling over a finite time scale of approximately $\epsilon^{-1}$.

Instead, the rigid locking of the dynamics to period doubling follows from the presence of long-range order in space that stems from spontaneously breaking $\mathbb{Z}_{2}$ Ising symmetry, whence "spatiotemporal" order [21]. This requires the Ising interactions $H_{z}$ to be the dominant part of the evolution during the first part of the drive. At any stroboscopic time, spins are locked into a "frozen" pattern in space so that $\left\langle Z_{i} Z_{j}\right\rangle$ is nonzero for arbitrarily large $|i-j|$ even in highly excited states (but can have a random, "glassy" sequence of signs as a function of $i, j$ ). This pattern then flips every period. Notably, the DTC phase is also stable to the addition of interactions that explicitly break Ising symmetry, such as longitudinal fields $H_{\text {int }}=\sum_{i} h_{i} Z_{i}$ $[20,21]$. In this case, the long-range spatial order follows from spontaneously breaking an emergent Ising symmetry [21]. This is a manifestation of the fact that the DTC phase is, in fact, stable to all weak perturbations of the Floquet unitary (1), including those not encapsulated by $H_{\text {int }}$ or $\epsilon$ - a feature termed absolute stability by a subset of the present authors [21].

In sum, the DTC is a robust, many-body phase of matter with spatiotemporal order (long-range order in space plus infinitely long-lived period doubling dynamics in time), realized in the intrinsically nonequilibrium setting of periodically driven, MBL quantum systems. Probing spatiotemporal order requires measuring site-resolved spatial correlations, e.g., $\left\langle Z_{i} Z_{j}\right\rangle$, and temporal autocorrelation functions, e.g., $\left\langle Z_{i}(n) Z_{i}\right\rangle$.

\section{B. First generation DTC experiments}

The DTC phase is particularly amenable to experimental detection due to its stability and its distinctive measurable dynamical signatures. Indeed, the theoretical prediction of this phase was rapidly followed by a pair of experiments, one on disordered trapped ions in one dimension [34] and the other on disordered nitrogen-vacancy (NV) centers in three-dimensional (3D) diamond [33]. An experiment using nuclear magnetic resonance (NMR) on a clean crystalline 3D solid followed soon after $[35,36]$. We refer to this set of experiments as "first generation" (FirstGen) time-crystal experiments.

Each of the FirstGen experiments simulates a model drive captured by Eq. (1). The experiments differ in various key details and, between them, realize a varied matrix of parameters, such as spatial dimension, range and type of interactions, nature of disorder, state preparation capabilities, microscopic controllability, etc. Each one represents an experimental tour de force, and manages to observe temporal signatures of DTC behavior (i.e., a signal locked at period doubling) over a finite extent in parameter space for the (finite) coherence time of the experiment. Despite the numerous differences between the platforms, the observed signatures look remarkably similar. However, despite these encouraging results, none of these platforms have all the ingredients needed for a genuine, asymptotic incarnation of the MBL DTC phase [22].

A key challenge for all three experiments lies in stabilizing MBL. Despite this, all three platforms still observe 
long-lived precursors of DTC order. This is because, even in cases where MBL is disallowed, it may nevertheless be possible to engineer a separation of scales such that thermalization happens on a parametrically slow scale - referred to as a "prethermal" regime in certain cases [48-52]. Specifically, the diamond NV center experiment [33] is incompatible with MBL because of its long-ranged interactions, but instead realizes a "critical TC" that thermalizes in a power-law slow fashion [53]. Likewise, the NMR setup [35] has no disorder and hence no MBL, and the long-lived signal therein was later explained as a prethermal phenomenon associated with a weakly broken global conservation law [54]. Finally, the trapped ion setup [34] is the smallest and most controllable, and has many of the necessary ingredients for realizing MBL; however, it was shown in Ref. [22] that, unexpectedly, the nature of disorder in the trapped ion TC experiment in Ref. [34] is also not sufficient for localization, and the signal observed therein also turned out to be of a prethermal rather than asymptotic nature. (However, as we discuss below, future iterations of the trapped ion experiment could, in principle, mitigate some of the issues of the first experiment.)

Despite not realizing an asymptotic MBL DTC, all three FirstGen experiments (and others [29], mentioned below) have greatly advanced our conceptual understanding of the DTC phase and led to new theoretical insights. These include the elucidation of a new mechanism for prethermalization [54] following the NMR experiment, and an understanding of the distinct types of disorder needed to stabilize MBL phases with distinct types of quantum order [22]. These insights have enabled us to formulate a detailed checklist of desired experimental capabilities for the next generation of DTC experiments. As an example, the eventual theoretical understanding of the FirstGen experiments as prethermal (or slowly thermalizing) phenomena - albeit of conceptually distinct genres - emphasizes that a key experimental challenge is to distinguish a genuine MBL DTC phase from a transient prethermal version. We emphasize that is an issue because of the finite times accessible to experiments rather than finite size (the diamond and NMR experiments have millions of spins so small systems sizes are not an issue, and slow prethermal dynamics stemming from large separations of parameter values arises even in infinitely large systems). In principle, the main difference between localized and prethermal DTCs lies in the lifetime of their quantum order: infinite for the former, transient for the latter. However, the ubiquity of environmental decoherence makes this distinction void in practice - measured DTC signals will be transient no matter what. Nevertheless, as we discuss below, finegrained measurements of spatially resolved observables on a variety of initial states can discriminate between prethermal and asymptotic TCs, even within finite experimental lifetimes.
TABLE I. Summary of experimental requirements for realizing and observing DTC spatiotemporal order, and the relative merits of different experimental platforms. The "double" check marks for the NV and NMR platforms in the "many-body" category are to emphasize that these setups, with $>O\left(10^{6}\right)$ constituents, are operating in the thermodynamic regime, at a size that is orders of magnitude larger than the trapped ion experiment (approximately 10 ions) and Sycamore (approximately 50 qubits).

\begin{tabular}{lcccc}
\hline \hline & \multicolumn{5}{c}{ Experiments } \\
\cline { 2 - 5 } Requirements & $\begin{array}{c}\text { NV } \\
\text { centers }\end{array}$ & $\begin{array}{c}\text { Trapped } \\
\text { ions }\end{array}$ & $\begin{array}{c}\text { NMR } \\
\text { crystal }\end{array}$ & Sycamore \\
\hline Definitional & & & & \\
$\quad$ Long coherence time & $\checkmark$ & $\checkmark$ & $\checkmark$ & $\checkmark$ \\
$\quad$ Many body & $\checkmark$ & $\sim$ & $\checkmark$ & $\checkmark$ \\
Stabilizing MBL & & & & \\
$\quad$ Short-range int. & $x$ & $?$ & $x$ & $\checkmark$ \\
$\quad$ Ising-even disorder & $\checkmark$ & $x$ & $x$ & $\checkmark$ \\
Detection & & & & \\
$\quad$ Site-resolved meas. & $x$ & $\checkmark$ & $x$ & $\checkmark$ \\
$\quad$ Varied initial states & $x$ & $\sim$ & $x$ & $\checkmark$ \\
\hline \hline
\end{tabular}

\section{Experimental checklist}

In all, the FirstGen DTC experiments, with their varied strengths and limitations, have been instrumental in distilling a checklist of experimental ingredients needed for the realization and detection of a bona fide DTC phase. These ingredients, and their presence or absence in the various experiments, are summarized in Table I and articulated in more detail below; these serve to achieve two intertwined goals.

(a) Realizing a genuine asymptotic MBL DTC phase, i.e., engineering all the theoretical criteria for achieving MBL and DTC order, so that an "ideal" experiment (without external decoherence) would observe an infinitely long-lived signal.This is a matter of principle - if internal decoherence (due to many-body quantum thermalization) in an ideal, noise-free incarnation of the platform destroys the signal at late times, then the system does not realize an asymptotic DTC phase (this is true of all FirstGen experiments). On the other hand, if the lifetime is predominantly limited by external decoherence, then this is an issue of engineering that will see sustained improvement with future hardware innovations.

(b) Detecting the spatiotemporal order that is a defining feature of the phase. This also entails experimentally discriminating between asymptotic (infinitely long-lived) and prethermal (transient) variants of DTCs, even within the constraints of environmental decoherence and finite experimental lifetimes.

We now enumerate six desired experimental capabilities, grouped in three broad categories. 


\section{Basic definitional requirements}

As mentioned earlier, a DTC phase is characterized by infinitely long lived, quantum-coherent oscillations in infinitely large, macroscopic many-body systems. While an actual experiment will always be of finite size with a finite coherence time, nontrivial realizations still require both of these to be sizeable, with room for parametric improvements with engineering advances. Thus, two basic requirements on the platforms are the following.

(i) Truly many body. The experimental systems should contain a number of qubits that does not qualify as "few body." While there is no sharp boundary between "few" and "many," it is clear that the NV and NMR experiments satisfy this requirement ( $>10^{6}$ qubits), while the trapped ion experiment (10 qubits) may be considered borderline - a few tens to hundreds of qubits would more comfortably fit the description. An added bonus is if the platform permits one to vary the system size, which would allow for finite-size scaling analysis of various order parameters. Another scenario ruled out by this requirement is that of effectively few-body systems where, despite a nominally large number of qubits, the dynamics becomes few body in a mean-field sense. Several recent TC experiments fall into this category [29,30,55,56], with Ref. [29] furnishing a particularly nice example using NMR on "star-shaped" molecules. We remark that this point is not about classical simulability, but specifically about physics. Time crystals are only nontrivial for macroscopic manybody systems; few-body systems exhibit special phenomena (e.g., recurrences) that do not scale to the many-body limit, and could prove confounding to the observation of the desired phenomenon.

(ii) Long coherence time. Experimental platforms aiming to exhibit dynamical phases clearly must be able to preserve quantum coherence for long enough, so that the underlying dynamical phenomena can be distinguished from short-time transients. Again, while there is no sharp boundary, revealing DTC order requires a coherence time of at least multiple tens to hundreds of Floquet cycles. We caution, however, that this may still not be enough to discriminate between MBL and prethermal TCs without using additional fine-grained probes [cf. points (v) and (vi) below]. All the FirstGen platforms had a lifetime of the order of 100 Floquet periods.

\section{Requirements for stabilizing $M B L$}

MBL is an essential ingredient for realizing a robust DTC phase in an extended region of parameter space, and in preventing periodic driving from heating the interacting system to infinite temperature. However, MBL is only stable under certain conditions sensitive to the range of interactions, and the scope for engineering disorder.

(iii) Short-ranged interactions. Long-ranged interactions are known to destabilize localization [57-59].
Interactions with strength scaling as $1 / r_{i j}^{\alpha}$ are incompatible with MBL if $\alpha<d$, where $d$ is the dimension of the system [57]. They are perturbatively compatible with MBL [60] if $\alpha>2 d$ [58]. Finally, the regime $d<\alpha<2 d$ is not fully understood in general; localization (or its absence) in this regime depends on the particular form of interactions present in specific Hamiltonians [58,59]. Out of the FirstGen experiments, the only one possibly satisfying the requirement of short-ranged interactions is that based on trapped ions $(d=1, \alpha \approx 1.51)$, but it is not presently settled whether the long-range Ising interactions therein are compatible with localization for the chosen value of $\alpha \approx 1.51$ [61]. This is indicated in Table I by a question mark. However, we note that, in principle, $\alpha$ is a tunable parameter in the trapped ion platform, and hence the trapped ion experiment could be repeated in the future with a value of $\alpha>2 d$. Both the diamond NV and NMR experiments have $d=\alpha=3$ and are thus not compatible with MBL.

(iv) Dominantly Ising interactions with Ising-even disorder. While stabilizing MBL generically requires disorder in the drive parameters, the nature of the disorder required to stabilize a MBL DTC is more specific: one requires strong disorder in Ising-even interactions $H_{z}=$ $\sum_{i j} J_{i j} Z_{i} Z_{j}$ [22] in a drive with dominantly Ising interactions of the form (1). If, instead, the only operators coupled to disorder are odd under the Ising symmetry $P_{x}=\prod_{i} X_{i}$ (as is the case, e.g., for on-site fields $H_{\text {int }}=h_{i} Z_{i}$ ), then this is not sufficient to stabilize MBL. This is because the Floquet evolution over two cycles, $U_{F}^{2}$, is only weakly disordered, and the dynamics is consequently not MBL. The effective disorder strength is weak because the Ising-odd disordered fields are "echoed out" by the approximate $\pi$ pulse, to leading order (see Appendix A for a discussion of this point). Of the FirstGen experiments, only the NV platform realizes Ising-even disorder due to the random position of NV centers in three-dimensional space; while this alone is not enough for MBL (because of the longrange interactions), the disorder still leads to algebraically slow themalization, giving a "critical time crystal" in the NV setup. The NMR system is clean and spatially ordered, and hence not localized. Finally, the trapped ion experiment features disorder only in Ising-odd longitudinal fields, while the Ising-even interactions are nonrandom and well approximated as $J_{i j} \sim J_{0} / r_{i j}^{\alpha}$. In a finite lattice the displacements $r_{i j}$ of the trapped ions (and thus the interactions $J_{i j}$ ) will include weak inhomogeneities due to the interplay of Coulomb interactions with the confining trap; however, these inhomogeneities are perfectly deterministic and reflection symmetric, and turn out to be insufficient to stabilize MBL [22]. In general, it is easier for many experimental setups to implement disorder in onsite fields rather than Ising couplings, and this requirement is a key engineering obstacle towards realizing DTCs on many such platforms, including in trapped ions. 


\section{Requirements for detection}

Finally, we turn to the requirements of unambiguously demonstrating the asymptotic DTC phase and distinguishing it from its transient prethermal cousins - even within the reality of finite experimental lifetimes.

The key discriminator is that MBL TCs show perioddoubled oscillations from all generic short-range correlated initial states, while prethermal TCs only show long-lived oscillations from certain special initial states. In addition, spatiotemporally resolved correlators show long-range order and period doubling in MBL TCs, while certain variants of prethermal TCs only show longlived oscillations in globally averaged observables but not site-resolved ones. Thus, studying varied initial states and making site-resolved measurements even for finite experimental times would distinguish between an asymptotic MBL DTC and all known alternate mechanisms that could support a prethermal DTC, as illustrated in Fig. 1.

In more detail, the key idea of prethermal dynamics is that, in a suitable reference frame, the system behaves for a long time as though it was governed by a static effective Hamiltonian (although the temperature of the state slowly increases en route to infinite temperature) $[48,49]$. If the effective Hamiltonian has an ordered phase below a critical temperature $T_{c}$, then a low-energy initial state would display quantum order for a long time, before eventually heating past $T_{c}$, thus causing the order to melt [50]. However, a high-energy initial state would not show any order, even for short times. Thus, practically, a useful discriminatory criterion is the dependence of the signal on the choice of initial state. In MBL DTCs there should be no strong dependence (as the whole spectrum is localized). On the other hand, prethermal DTCs associated with symmetry breaking display long-lived oscillations for lowtemperature ordered states but not for others [Figs. 1(a) and $1(b)]$.

Separately, another mechanism for prethermalization is the emergence of a quasiconserved quantity associated to an approximate symmetry of the prethermal Hamiltonian $[49,54]$. This mechanism for slow thermalization can be at play even for very high-temperature initial states. In this case, measurements of global observables such as the total magnetization are at risk of detecting the slow relaxation of a quasiconserved quantity rather than the DTC pattern of spatiotemporal order. However, measuring siteresolved correlations can distinguish between prethermal U(1) DTCs and MBL DTCs [Figs. 1(a) and 1(c)]. Thus, the following conditions are required.

(v) Widely tunable initial states. To distinguish localized and prethermal DTCs within a finite experimental lifetime, one needs to test a variety of initial states (prethermal DTCs are highly sensitive to the choice unlike MBL DTCs). In practice, the ability to prepare any computational basis state, i.e., product states in the $z$ basis,
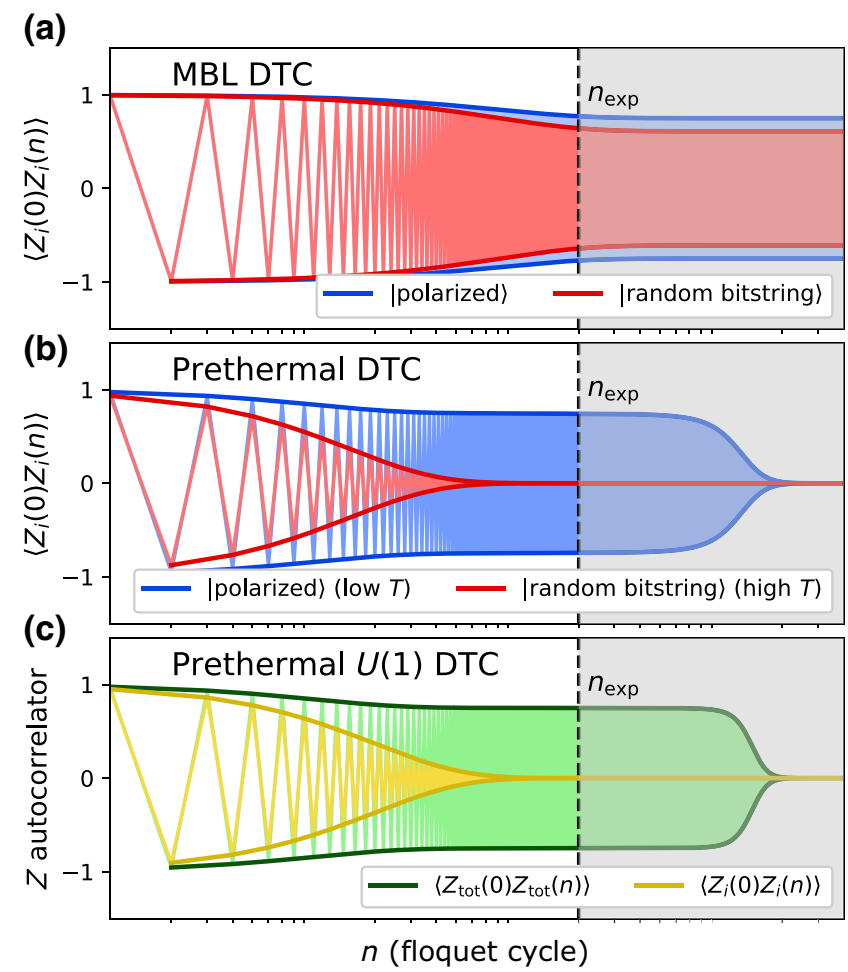

FIG. 1. Illustrative sketch for distinguishing MBL and prethermal DTCs, even with access to only finite experimental coherence times (vertical dashed line, $n_{\exp }$ ). (a) In a MBL DTC, local autocorrelators remain large for all initial product states in the $Z$ basis. (b) In a prethermal DTC, low-temperature initial states have long-lived autocorrelators whose lifetime may exceed the experimental coherence time. However, generic bitstring initial states (high temperature) decay quickly: the dependence on initial states-visible within experimental time scales $n_{\exp }$ - is a signature of prethermalization. (c) In a $U(1)$ prethermal DTC, the total magnetization $Z_{\text {tot }}=\sum_{i} Z_{i}$ is nearly conserved (for all initial states, including high-temperature ones). However, local operators $Z_{i}$ decay quickly: the $U(1)$-prethermal behavior is revealed through site-resolved measurements.

would be enough. This cannot be done on platforms that only allow for the preparation of special initial states, such as fully polarized ones. Of the FirstGen platforms, only the trapped ion experiment has the capability to widely vary initial states, although this was not fully explored in Ref. [34]. The experiment only considered two initial states: a fully polarized state, $|0\rangle^{\otimes L}$, and a state polarized on the left and right halves, $|0\rangle^{\otimes L / 2}|1\rangle^{\otimes L / 2}$. However, polarized or near polarized states are maximally ineffectual at distinguishing between MBL and prethermal dynamics $[22,54]$. Because the trapped ion experiment has longrange interactions, the effective Hamiltonian governing the prethermal dynamics can have an Ising symmetry breaking transition at a finite temperature $T_{c}$ even in one dimension, and near polarized states are in the low-temperature sector of the effective Hamiltonian. Indeed, detailed numerical 
simulations of the trapped ion experiment on a wider class of initial states found strong initial state dependence, with the DTC signal decaying much more rapidly for randomly picked computational-basis states, consistent with prethermal DTC order [22]. Separately, a different mechanism for prethermalization entails the long-lived quasiconservation of a global operator such as the total magnetization. Again, polarized initial states have large total magnetization and can show slow dynamics due to the quasiconservation law, while randomly picked $z$ product states would not.

(vi) Site-resolved measurements. Detecting genuine spatiotemporal order requires measuring site-resolved spatial correlation functions of the form $\left\langle Z_{i} Z_{j}\right\rangle$, in addition to temporal autocorrelators. This capability to locally probe individual qubits is also necessary for distinguishing MBL TCs from prethermal variants involving global quasiconservation laws. For instance, the NMR experiment operates in an extremely hot regime, with very high temperature initial states that would be well above the ordering temperature $T_{c}$ of the effective Hamiltonian; but these can still show slow dynamics in global observables that couple to a quasiconservation law, such as the total magnetization [54]. In contrast, local autocorrelators would show a fast decay in this regime. In contrast, site-resolved autocorrelators show oscillations forever in a MBL TC. The NMR and NV center experiments (which involve $>10^{6}$ qubits) are limited to probing spatially averaged quantities such as the total magnetization $\sum_{i} Z_{i}$, which do not provide the necessary resolution. Among the FirstGen experiments, only the trapped ion experiment satisfies this requirement. Table I summarizes the matrix of experimental desiderata and their availability in different FirstGen experiments.

We now turn to how the next generation (NextGen) of quantum simulators - such as the already operational Google Sycamore processor - can be programmed to realize all these ingredients in turn, and hence to furnish the first bona fide realization of the time-crystal phase. We should note that, while the trapped ion experiment has not yet demonstrated a MBL DTC phase, it may be possible for future iterations of this platform to do so. The key engineering challenges entail scaling up the system to suitably larger numbers of ions, and adding uncorrelated disorder in the Ising couplings $J_{i j}$ (which is possible, in principle, with extensively many tuning knobs $[62,63])$. These are achievable given enough time and effort. Likewise, quantum simulators using Rydberg or dressed Rydberg atoms meet almost all the desired criteria, and are currently limited only by their coherence time [64]. Future improvements will no doubt also enable the observation of such phenomena on this versatile platform. However, as we demonstrate next, currently existing capabilities in the Sycamore device already satisfy all the desiderata and, indeed, the platform seems tailor made for this application.

\section{NEXT GENERATION: REALIZING A DTC ON THE SYCAMORE PROCESSOR}

NextGen programmable quantum simulators are designed with quantum computing applications as a major drive. These applications happen to require many of the items of the above checklist. The preparation of arbitrary computational-basis states and the capability for siteresolved readout are both key ingredients for quantum computing [9], so it is fair to assume their availability on a NISQ device, up to small control and measurement errors. Moreover, these devices are designed to implement quantum circuit elements that are typically one- and two-qubit gates, which in the quantum manybody language means on-site fields and nearest-neighbor interactions. While the selective realization (elimination) of short-ranged (long-ranged) couplings is an engineering challenge in all quantum computing platforms (currently addressed with varying degrees of accuracy in each one, and sure to see sustained effort in the future), it is fair to assume that on near-term digital quantum simulators crosstalk between distant qubits will be limited, and the dominant interactions will be between neighboring qubits. Such finite-range interactions are much more suitable for MBL compared to the power-law decaying couplings native to many platforms [65]. Thus, shortranged interactions [requirement (iii)], site-resolved measurements [requirement (vi)], and tunable initial states [requirement (v)] are all at our disposal, within reasonable levels of approximation. Moreover, as these devices enter the 50-to-200-qubit NISQ regime [3], they can be safely regarded as legitimate quantum many-body systems [requirement (i)].

According to the checklist in Sec. II C, the last two points to be addressed are (a) whether the coherence times are long enough, given the eponymous noise inherent to NISQ devices and (b) whether the devices can implement a kicked Ising drive similar to that in Eq. (1), with disorder in the Ising couplings, $J_{i j}$. While a universal fault-tolerant quantum computer can, of course, realize any drive with any set of couplings $[12,66]$, present-day NISQ devices may present obstructions due to their finite coherence time. Again, we are motivated by near-term applications that are immediately and naturally realizable on these platforms (as opposed to universally and asymptotically). To address these points in a more specific way, we focus on Google's Sycamore processor for the remainder of this work. In Sec. III A we lay out the details of implementing the Floquet DTC as a quantum circuit with gates available on Sycamore, while in Sec. III B we map out the phase diagram of this circuit model and present several diagnostics of the MBL DTC phase. All of the analysis for now assumes an "ideal," i.e., decoherence-free realization; the analysis of noise that informs the coherence time is presented in Sec. IV. 


\section{A. Floquet DTC circuit on Sycamore}

We begin by noting that the Floquet unitary evolution operator for the canonical model of a DTC, Eq. (1), can be naturally written as a sequence of gates when $H_{\text {int }}=0$, and when the $J_{i j}$ couplings are limited to nearest neighbors. We confine the dynamics to a one-dimensional system, where the existence of MBL and thus of the DTC phase is on firmest ground $[42,65]$. In this case, one first acts with a layer of Ising gates $e^{-i J Z Z}$ on the even bonds of the 1D subsystem, then a layer of Ising gates on the odd bonds, and then a layer of single-qubit $X$ rotations, $e^{-i g X}$ :

$$
\begin{aligned}
U_{F} & =e^{-i g \sum_{i} X_{i}} e^{-i \sum_{i} J_{i} Z_{i} Z_{i+1}} \\
& =\prod_{i} R_{i}^{x}(2 g) \prod_{i} e^{-i J_{2 i-1} Z_{2 i-1} Z_{2 i}} \prod_{i} e^{-i J_{2 i} Z_{2 i} Z_{2 i+1}}
\end{aligned}
$$

with $R_{i}^{x}(\alpha)=e^{-i \alpha X_{i} / 2}$ a single-qubit $X$ rotation. This model has Ising symmetry and is exactly solvable, being mappable to free fermions. In this limit, the system is in the DTC phase (with period-doubled dynamics and spontaneously broken Ising symmetry) as long as the average $J$ couplings obey [19]

$$
\left|\overline{J_{i}}-\frac{\pi}{4}\right| \leq g-\frac{\pi}{4}
$$

(one can take $g, \overline{J_{i}} \in[0, \pi / 2]$ without loss of generality as the phase diagram repeats symmetrically outside this square). As mentioned earlier, the DTC phase persists for a finite region in parameter space surrounding $g=\pi / 2$, even upon perturbing the drive in Eq. (2) with generic interactions to make the model nonintegrable, as long as the disorder in $J_{i}$ is strong enough to stabilize MBL.

On the Sycamore chip, a unitary evolution close to Eq. (2) can be straightforwardly implemented. Singlequbit $X$ rotations $R_{i}^{x}$ are readily available [5]. For the two-qubit interaction, the Sycamore device allows implementation of a continuously parameterized family of highfidelity gates of the form $[67,68]$

$$
G_{1,2}=R_{1}^{z}\left(h_{a}\right) R_{2}^{z}\left(-h_{a}\right) F_{1,2}(\theta, \phi) R_{1}^{z}\left(h_{b}\right) R_{2}^{z}\left(h_{c}\right),
$$

where $R_{i}^{z}(\alpha)=e^{-i \alpha Z_{i} / 2}$ is a single-qubit $Z$ rotation, the $h$ angles result from the frequency excursion of the single qubits during the interaction [69], and $F$ is the "fermionic simulation" two-qubit gate [70],

$$
\begin{aligned}
& F_{1,2}(\theta, \phi) \\
& \quad=\exp \left(-i \frac{\theta}{2}\left(X_{1} X_{2}+Y_{1} Y_{2}\right)-i \phi \frac{Z_{1}-I}{2} \frac{Z_{2}-I}{2}\right),
\end{aligned}
$$

defined by an "isWAP angle" $\theta$ and a "controlled-phase angle" $\phi$. The latter provides the crucial ingredient for the
Floquet DTC unitary: the two-qubit Ising coupling $e^{-i J Z Z \text {, }}$ with the identification $J \equiv \phi / 4$.

The remaining terms in Eq. (4), i.e., the iswaP angle $\theta$ and the single-qubit $Z$ rotations (coming both from $F$ and from the $h$ angles), represent deviations away from the solvable limit in Eq. (2), but these deviations can be controlled and manipulated rather straightforwardly. Specifically, the angles $\theta_{i j}$, one for each coupler in the Sycamore chip, can be independently tuned to arbitrary values (including zero) within calibration accuracy. For the purpose of this paper, we sample each $\theta_{i j}$ out of a normal distribution with variable mean $\bar{\theta}$ and standard deviation $\Delta \theta=\pi / 50$, representing a gate calibration error of a few degrees $\left(\pi / 50 \mathrm{rad}=3.6^{\circ}\right)$, a deliberately conservative upper bound. The "extra" single-qubit $Z$ rotations can also be tuned and canceled "by hand" (within calibration accuracy) with active $Z$ rotations of appropriate angles on each qubit before and after each application of $G$; see Fig. 2(a). The result is a modified gate

$$
\begin{aligned}
\widetilde{G}_{i, j}= & R_{i}^{z}\left(\delta h_{a}^{i j}\right) R_{j}^{z}\left(-\delta h_{a}^{i j}\right) e^{-i \theta_{i j}\left(X_{i} X_{j}+Y_{i} Y_{j}\right) / 2-i \phi_{i j} Z_{i} Z_{j} / 4} \\
& \times R_{i}^{z}\left(\delta h_{b}^{i j}\right) R_{j}^{z}\left(\delta h_{c}^{i j}\right),
\end{aligned}
$$

where the $\delta h$ are small residual rotation angles, taken to be normal random variables of standard deviation $\Delta h=$ $\pi / 50$. Note that the nonzero $\Delta h, \bar{\theta}$, and $\Delta \theta$ make the model genuinely interacting and nonintegrable; the $\Delta h$ terms also break the Ising symmetry. Both effects are necessary for a nontrivial demonstration of the stability of the phase. Thus, even as calibration errors continue to improve, these deviations can and should be deliberately included for a nontrivial demonstration of the phase. We have explicitly verified by numerical diagonalization that $\Delta h=\Delta \theta=\pi / 50$ is large enough to visibly break integrability even when $\bar{\theta}=0$.

With the $\widetilde{G}$ gate defined above, it is now straightforward to define our model Floquet circuit,

$$
U_{F}=\prod_{i} R_{i}^{x}(2 g) \prod_{i} \widetilde{G}_{2 i-1,2 i} \prod_{i} \widetilde{G}_{2 i, 2 i+1},
$$

sketched in Fig. 2(b). This represents a generically perturbed and nonintegrable variant of the solvable model in Eq. (2). Single-qubit rotations are widely and easily tunable on Sycamore, allowing for arbitrary values of the $\hat{x}$ rotation angle $2 g$ (or, equivalently, the $\pi$-pulse imperfection $\epsilon=\pi-2 g$ ). The two-qubit gates act, in turn, on the even and odd bonds along a one-dimensional path through Sycamore, such as that sketched in Fig. 2(c). All the parameters specifying the individual $\widetilde{G}_{i j}$ gates $\left(\phi_{i j}, \theta_{i j}\right.$, $\delta h_{a, b, c}^{i j}$ ) are drawn randomly for each gate (one per spatial bond), but are time independent: all these choices are fixed once per realization, and then repeated in time so as to define an ideal time-periodic (Floquet) model [71]. 
(a)
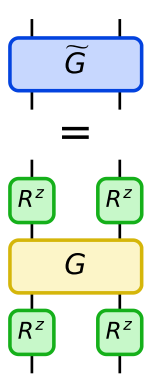

(c)

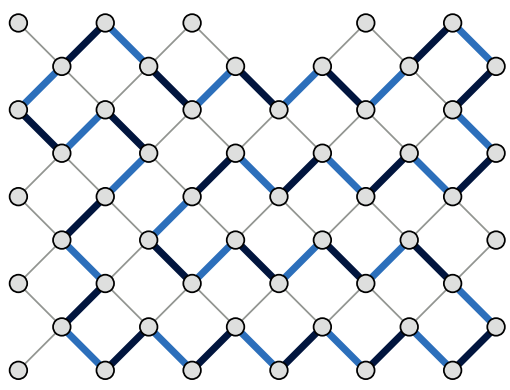

(b)

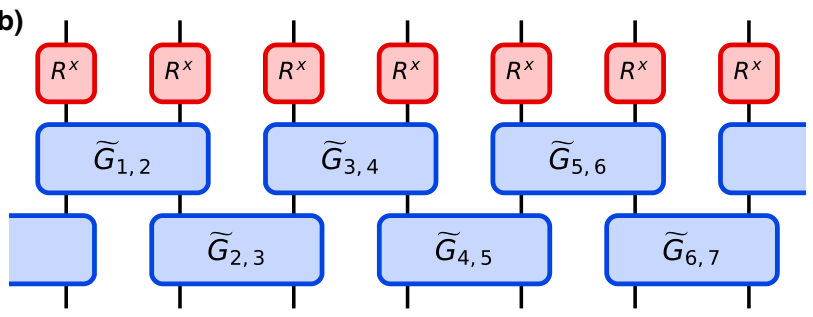

FIG. 2. Simulating a 1D Floquet DTC on the Sycamore chip. (a) Modified gate $\widetilde{G}$ in terms of the native gate $G$ and single-qubit $Z$ rotations. (b) Circuit for the DTC Floquet unitary: each Floquet cycle acts with $\widetilde{G}$ on each pair of neighboring qubits, followed by single-qubit $X$ rotations, as depicted. (c) A closed loop through the Sycamore chip, simulating a 1D system. During each cycle, $\widetilde{G}$ gates act first on the blue bonds, then on the black bonds. All other bonds remain idle during the dynamics.

Again, we chose to use a one-dimensional path through Sycamore rather than the full 2D array of couplers in order to remain within the territory where MBL and the DTC phase are firmly established on theoretical grounds. However, we note the extreme flexibility of this platform in potentially choosing different geometries - e.g., 1D paths of different lengths, with open or periodic boundary conditions, or 2D patches of various shapes - all on the same chip, simply by selecting which couplers to activate and which to leave idle during the dynamics.

Having discussed the parameters $g, \theta_{i j}, \delta h_{a, b, c}^{i j}$ above, we now turn to the $\phi_{i j}$ angles, which set the strength of the $Z Z$ coupling and address the final requirement of Isingeven disorder. From an engineering perspective, two-qubit gates are generally more demanding than single-qubit rotations: each distinct gate acting on a given bond $\langle i, j\rangle$ must be calibrated individually [67]. The phases $\phi_{i j}$ are thus drawn randomly from a discrete set of $M$ values $(M \lesssim 10$ appears realistic in the near term), rather than a continuous distribution as is usually assumed in studies of MBL. This is because choosing gate parameters from a continuum would require one to calibrate each gate in the circuit for each distinct disorder realization, making the process highly impractical. In contrast, it is vastly easier to calibrate $M$ gates per bond at the beginning (so approximately $L M$ distinct gates in total), and then use these to generate a virtually infinite (approximately $M^{L}$ ) number of disorder realizations.

In this work we choose the discrete set of disordered couplings to be

$$
\{\bar{\phi}+W \cos [\pi m /(M-1)]: m=0, \ldots, M-1\},
$$

where $\bar{\phi}$ sets the average coupling and $W$ the disorder strength. The use of a nonlinear function ensures that there are incommensurate spacings between the different phases $\phi_{i j}$, thus limiting the effect of accidental resonances [72]; the choice of $\cos (x)$ is otherwise arbitrary and is expected to yield generic results. For specificity, in the following we fix the average controlled-phase angle to $\bar{\phi}=\pi$ corresponding to $\bar{J}=\pi / 4$. This choice is at the center of the DTC phase in the noninteracting model, and allows for the widest range of rotation angles $g$ [cf. Eq. (3)]. The disorder strength is set to $W=\pi / 2$; this is fairly strong while also ensuring that all the $\phi$ angles are far from 0 (where the experimental implementation could be problematic in some cases [68]). Finally, we set $M=8$ based on numerical results obtained via full diagonalization of the Floquet unitary $U_{F}$ that indicate that $M=8$ disorder values are sufficient to qualitatively replicate the continuous disorder $(M \rightarrow \infty)$ case.

The quantum circuit so defined captures all the crucial aspects of the canonical Floquet DTC, Eq. (1), in a "Trotterized" form. It differs from the solvable limit, Eq. (2), in specific ways: the nonzero isWAP angles $\theta_{i j}$ introduce interactions and make the model nonintegrable; the nonzero longitudinal fields, $\Delta h$, also add interactions and weakly break the Ising symmetry; and finally the disorder in the $\phi_{i j}$ couplings is discrete rather than continuous.

In the following we confirm that these do not destroy the DTC phase, as expected from its absolutely stable nature [21]. By varying $g$ and $\bar{\theta}$, with all other parameters fixed as described above, we obtain a phase diagram for the model circuit, shown in Fig. 3. This was obtained by combining various phase diagnostics, discussed in the next section. It includes two MBL phases for sufficiently weak $\bar{\theta}$ : a DTC phase near $g=\pi / 2$ (corresponding to an imperfect $\pi$ flip), and a paramagnetic phase near $g=0$. These are separated by a large thermal region, which expands as the interaction strength $\bar{\theta}$ is increased, eventually destroying both MBL phases for $\bar{\theta} \gtrsim \pi / 8$. The next section presents a detailed discussion of the diagnostics used to obtain this phase diagram and to detect the different phases in an experimental setting.

\section{B. Diagnostics of the MBL DTC phase}

Nonequilibrium phases and phase transitions are understood as eigenstate phases [18,73-75]; their theoretically sharpest diagnostics involve properties of the many-body eigenspectrum and of individual many-body eigenstates of 


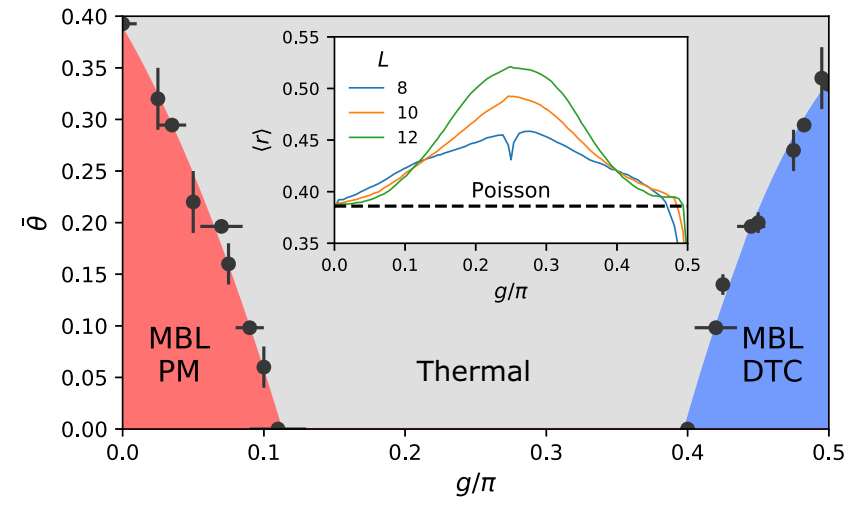

FIG. 3. Phase diagram of the circuit, Eq. (7), as a function of the pulse parameter $g$ and the average iswAP angle $\bar{\theta}$. The phase boundary is based on finite-size crossing points (black dots) of the level spacing ratio, computed numerically for systems of $8 \leq$ $L \leq 12$ qubits. Inset: level spacing ratio $\langle r\rangle$, Eq. (9), versus $g$ on the $\bar{\theta}=0$ cut. The value of $\langle r\rangle$ is averaged over eigenstates and over between 400 and 4000 realizations of disorder (depending on $L$ ).

the Floquet unitary $U_{F}$, which change in a singular manner across phase boundaries. While theoretically useful, these eigensystem diagnostics are not directly accessible to experiment, and their numerical exploration is limited to the small sizes amenable to exact diagonalization of $U_{F}$. Fortunately, these diagnostics translate to distinctive measurable signatures in dynamics from generic computational-basis initial states, that are both observable in experiment and accessible to numerics for much larger sizes.

We now present various eigenspectrum and dynamical diagnostics for identifying both MBL and the DTC order, which were used to derive a phase diagram for the model presented in the previous section.

Level repulsion. Many-body localization, aside from its dynamical signatures in the form of a persistent memory of initial conditions, is characterized by the absence of repulsion between quasienergy levels in the spectrum of $U_{F}$. The eigenvalues of $U_{F}$ are phases $\left\{e^{-i E_{n}}\right\}$; these can be used to obtain the quasienergies $\left\{E_{n}\right\}$, defined modulo $2 \pi$. The statistics of quasienergy levels has been a powerful tool in the numerical study of MBL on finite systems, in particular the level-spacing ratio [76]:

$$
r=\frac{\min \left(\delta_{n}, \delta_{n+1}\right)}{\max \left(\delta_{n}, \delta_{n+1}\right)}
$$

with $\delta_{n}=E_{n+1}-E_{n}$, the $n$th spacing between the quasienergies of $U_{F}$. In a MBL phase, the value of $\langle r\rangle$ averaged over eigenstates and disorder realizations approaches the Poisson value $\langle r\rangle_{\text {Poisson }} \simeq 0.39$ with increasing system size, reflecting the lack of level repulsion that arises from localization. In an ergodic phase it should instead approach the Gaussian unitary ensemble (GUE) value $\langle r\rangle_{\mathrm{GUE}} \simeq 0.60$, characteristic of random-matrix behavior [77]. Finite-size scaling of this quantity across different cuts in parameter space is used to map out the phase diagram in Fig. 3. The inset displays one such cut, at $\bar{\theta}=$ 0 , with two crossings separating the thermal phase $(\langle r\rangle$ increasing with $L)$ from the two MBL phases ( $\langle r\rangle$ decreasing with $L$ ). Note that the dip below the Poisson value near $g=\pi / 2$ is a finite-size effect due to the restoration of the Ising symmetry at $g=\pi / 2$, where the $h$ fields are exactly "echoed out" over two periods.

Real-time oscillations. The level spacing ratio distinguishes between MBL and thermal phases, but not between different MBL phases. To do this, we need to consider specific features of the quantum order inherent in a MBL DTC. The hallmark of a DTC is spatiotemporal order: infinitely long-lived period-doubled oscillation of spins, in conjunction with long-range glassy order in space. This is encoded in the behavior of a two-point correlation function $[19,21]$

$$
C_{i j}(n)=\left\langle Z_{i}(0) Z_{j}(n)\right\rangle \propto(-1)^{n} s_{i j}
$$

at late times, where $n$ counts Floquet cycles and $s_{i j}$ encodes the "glassy" spatial order (i.e., is nonzero, but may have random sign as a function of $i$ and $j$ ). This means that memory of an initial glassy configuration is preserved forever, with the configuration itself flipped at every cycle. Starting from a computational basis state $|\psi(0)\rangle=$ $|\boldsymbol{\sigma}\rangle\left(\sigma \in\{0,1\}^{L}\right)$, the statement in Eq. (10) simplifies to $\left\langle Z_{j}(n)\right\rangle \propto(-1)^{n}\left\langle Z_{j}(0)\right\rangle$ : each spin gets flipped at every cycle, while maintaining a finite fraction of its initial (maximal) polarization. In contrast, a MBL paramagnet will retain memory of the initial configuration, but the spins do not get flipped.

We perform exact numerical simulations of time evolution (via sparse matrix-vector multiplication) under the circuit, Eq. (7), on systems of up to $L=22$ qubits starting from various computational basis states (ranging from polarized states to pseudorandom bitstrings). Representative plots for all three phases are shown in Figs. 4(a)-4(c) for $\bar{\theta}=0$ and one value of $g$ in each phase. We compute and plot $C(n)=(1 / L) \sum_{i} \overline{C_{i i}(n)}$ that is the spatially resolved autocorrelator, Eq. (10), averaged over all sites $i$ and over at least $10^{3}$ disorder realizations. In the DTC phase, all initial states show a persistent period-doubled DTC signal $C(n) \propto(-1)^{n}$ up to at least $n_{\max }=10^{4}$ Floquet cycles [Fig. 4(a)]. In contrast, the MBL paramagnetic phase near $g=0$ shows a persistent signal $C(n)$, but at frequency $\omega=0$ rather than $\omega=\pi$ [Fig. 4(c)]. The large steady signal for a wide range of choices in initial states is a signature of MBL DTCs, which distinguishes them from prethermal DTCs. For example, a similar numerical simulation of autocorrelators in the trapped ion experiment sees strong state-to-state dependence, with $C(n)$ quickly decaying for most initial states [22]. Finally, the behavior of both 

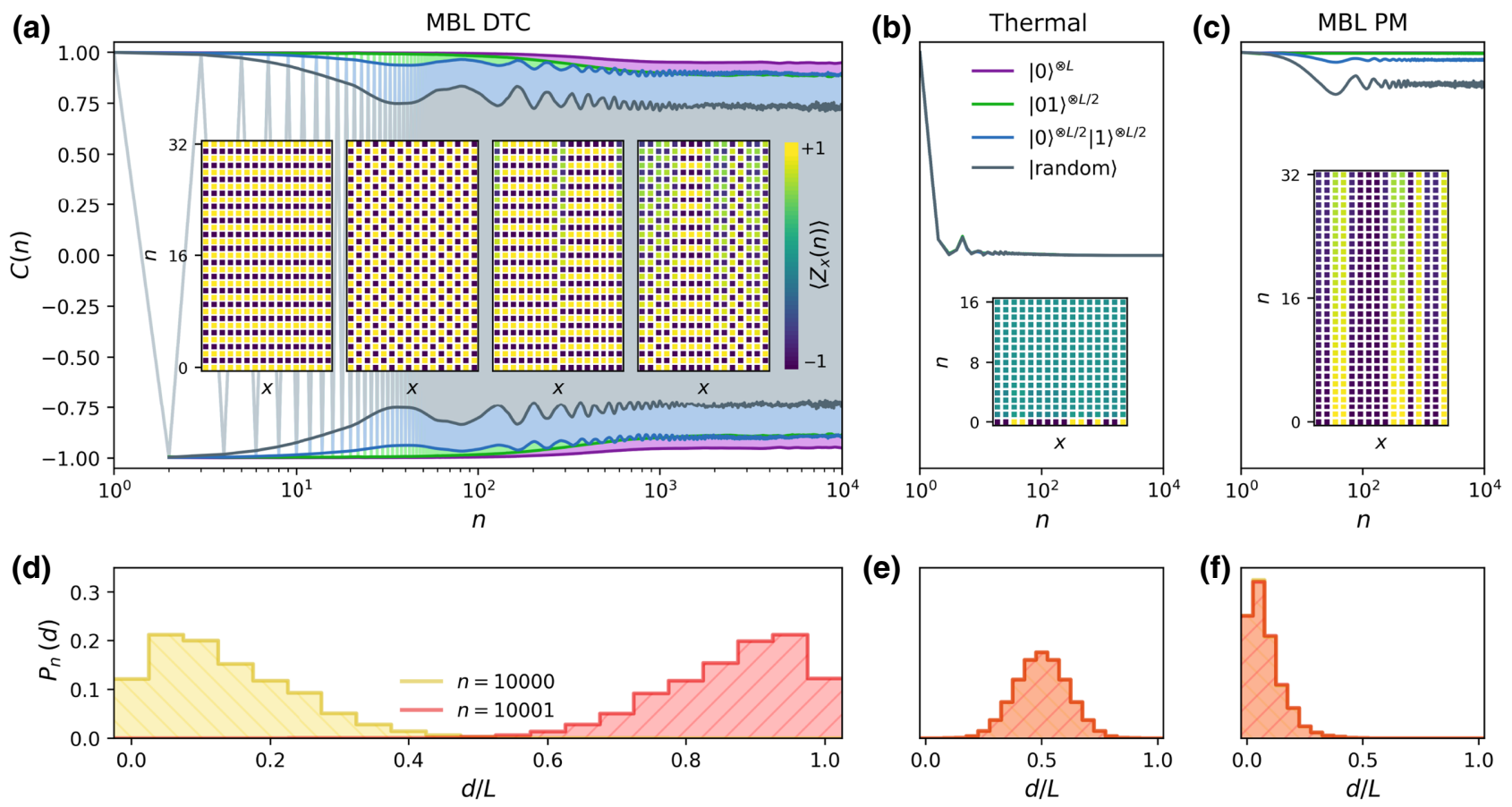

FIG. 4. Dynamics of the ideal (noise-free) circuit in the MBL DTC $(g / \pi=39 / 80)$, thermal $(g / \pi=19 / 80)$, and MBL paramagnetic $(g / \pi=1 / 80)$ phases $(\bar{\theta}=0)$. (a)-(c) Position- and disorder-averaged temporal autocorrelator $C(n)$ starting from various initial bitstring states for $L=20$ qubits. In the DTC phase the envelopes at even and odd times are highlighted; the signal oscillates stroboscopically between these two envelopes (lighter curves). Insets: space-time color plots of expectation values $\left\langle Z_{x}(n)\right\rangle$ for $L=16$ qubits. (d)-(f) Disorder-averaged probability distribution of the Hamming distance $d$ from the initial bitstring in two consecutive Floquet cycles at late time, $n=10^{4}$, for $L=20$ qubits.

MBL phases should be contrasted with that of the thermal phase [Fig. 4(b)] where the autocorrelator $C(n)$ quickly decays to zero for all initial states.

The insets for panels (a)-(c) in Fig. 4 show space-time color plots of $\left\langle Z_{i}(t)\right\rangle$, visually depicting the oscillating glassy order in the MBL DTC, frozen memory in the MBL paramagnet, and rapid thermalization in the thermal phase. Importantly, measuring such site-resolved space-time correlators for a wide range of initial states is well within the existing capabilities of the Sycamore device. As discussed in Sec. II C, such measurements are essential for a detection of the spatiotemporal order that defines the MBL DTC, and for distinguishing between MBL DTCs and prethermal variants.

Frequency-space peaks. The real-time dynamics can also usefully be examined in frequency space, and used to probe how the DTC order melts and gives way to a thermal phase as the $\pi$-pulse imperfection $\epsilon=\pi-2 g$ is increased [19]. Figure 5(a) shows data obtained from dynamics simulations of $L=14$ to 20 qubits at several values of the pulse parameter $g$ between $g=\pi / 2$ (perfect $180^{\circ}$ pulse, center of the DTC phase) and $g=\pi / 4$ (center of the thermal phase). The position- and disorder-averaged autocorrelator $C(\omega)$ [obtained from Fourier transforming the real-time signal $C(n)$ collected out to $n_{\max }=10^{4}$ ] shows a peak at $\omega=\pi$ in the DTC phase, as expected; its height drops smoothly as one exits the phase [Fig. 5(a)]. While this is expected to sharpen with increasing system size, the finitetime limitation turns this into a smooth crossover [Fig. 5(a) inset]. Such an analysis can, of course, also be done with experimentally measured dynamical signals.

Given that real-time dynamics simulations are inevitably limited to finite time $n$, a useful complementary perspective is achieved by examining spectral functions, where - at the expense of more severe finite-size limitations - we can effectively probe infinitely long times by a full diagonalization of the Floquet unitary $U_{F}$. The period-doubled behavior in Eq. (10) corresponds to a sharp delta-function peak at frequency $\omega=\pi$ in the spectral function

$$
\mathscr{C}_{i j}(\omega)=\frac{1}{2^{L}} \sum_{\mu, \nu}\left\langle\mu\left|Z_{i}\right| \nu\right\rangle\left\langle\nu\left|Z_{j}\right| \mu\right\rangle \delta\left(E_{\mu}-E_{\nu}-\omega\right),
$$

where $\mu, v$ label the eigenstates of the Floquet unitary $U_{F}$ and the $E_{\mu}$ are its quasienergies, i.e., $U_{F}|\mu\rangle=e^{-i E_{\mu}}|\mu\rangle$. This function represents a Fourier transform of the autocorrelator, Eq. (10), over infinite stroboscopic times and averaged over all initial states. It was used in conjunction with the level statistics to map the phase diagram in Fig. 3, as described below. 

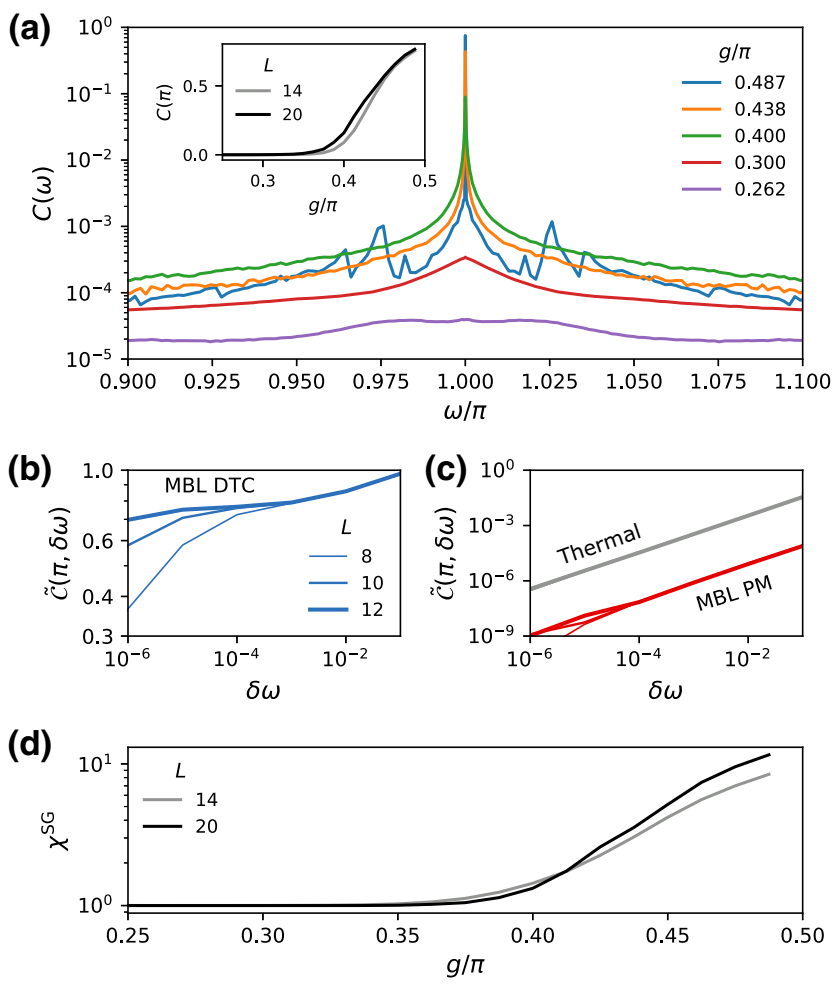

FIG. 5. Other diagnostics of the DTC order. (a) Fourier transform of the temporal autocorrelator, $C(\omega)$, averaged over position and disorder, for several values of $g$ spanning the DTC and thermal phases. Data from dynamics simulations of $L=18$ qubits starting from a fixed bitstring state and evolving to $n_{\max }=$ $10^{4}$ Floquet cycles. Inset: height of the $\omega=\pi$ peak as a function of $g$. (b),(c) Spectral function $\mathscr{C}(\pi, \delta \omega)$ [see Eq. (12)] from exact diagonalization of $U_{F}$ on small sizes, averaged over disorder. The DTC phase develops a plateau for $\delta \omega \rightarrow 0$ corresponding to a delta-function $\pi$ peak in the Fourier response, while in the thermal and MBL PM phases we find that $\mathscr{C}(\pi, \delta \omega) \sim \delta \omega$. (d) Spin glass order parameter $\chi^{\mathrm{SG}}$ evaluated at late times, $n_{\max } / 2 \leq$ $n \leq n_{\max }$, from dynamics simulations as in (a). A crossing for increasing system size indicates a transition consistent with the phase boundary in Fig. 3 at $\bar{\theta}=0$.

In a finite-size system, the spectral function $\mathscr{C}_{i j}(\omega)$ must be regularized by integrating over a finite frequency window $\delta \omega$,

$$
\tilde{\mathscr{C}}_{i j}(\omega, \delta \omega) \equiv \int_{\omega-\delta \omega}^{\omega+\delta \omega} d \omega^{\prime} \mathscr{C}_{i j}\left(\omega^{\prime}\right)
$$

A delta-function peak $\mathscr{C}_{i j}(\omega) \sim \delta(\omega-\pi)$ in the infinitesize limit translates to a finite limit

$$
\lim _{\delta \omega \rightarrow 0} \tilde{\mathscr{C}}_{i j}(\pi, \delta \omega)=\mathrm{const} \neq 0
$$

as opposed to the generic non-DTC behavior $\tilde{\mathscr{C}}_{i j}(\omega, \delta \omega) \sim$ $\delta \omega^{\gamma}, \gamma>0$ as $\delta \omega \rightarrow 0$. Figures 5(b) and 5(c) show numerical results for $\tilde{\mathscr{C}}(\omega=\pi, \delta \omega)$ at representative points in the three phases. The onset of a plateau is clearly visible for increasing system size in the DTC phase, indicating the formation of a delta-function peak in $\mathscr{C}(\omega)$ at $\omega=\pi$. Both the thermal and MBL paramagnetic phases instead obey the scaling $\tilde{\mathscr{C}}(\omega, \delta \omega) \sim \delta \omega \rightarrow 0$ as $\delta \omega \rightarrow 0$.

Glassy spatial order. As discussed already, a key feature of the DTC phase is long-range spatial "spin-glass" order that stems from spontaneously breaking an (emergent) Ising symmetry $[19,21]$. This can be detected from long-range spatial correlation functions measured in the many-body eigenstates of the Floquet unitary (or, equivalently, from nonzero mutual information between distant subregions of the eigenstates [20]). It can also be detected in dynamics through autocorrelators of the form Eq. (10).

Here we use a classic diagnostic of spin glasses related to the Edwards-Anderson order parameter [78]:

$$
\chi^{\mathrm{SG}}=\frac{1}{L} \sum_{i, j}\left\langle\psi\left|Z_{i} Z_{j}\right| \psi\right\rangle^{2} .
$$

This quantity is extensive in a phase with glassy order (where all $L^{2}$ items in the sum are finite); otherwise, it is of order 1 (with only the $i=j$ contributions being significant). It can be examined in the many-body eigenstates of a Hamiltonian or of $U_{F}[19,79]$, and its finite-size scaling provides yet another mechanism to deduce the phase diagram in Fig. 3.

Importantly, in a platform such as Sycamore with full spatial resolution, this quantity can also be examined dynamically starting from varied initial states. In Fig. 5(d), $\chi^{\mathrm{SG}}$ (averaged over late times and disorder realizations) is plotted as a function of $g$ for $\bar{\theta}=0$, and clearly shows a crossing with increasing system size, at a value of $g$ consistent with the phase boundary in Fig. 3. Note that the effective system size probed on Sycamore can be easily varied by choosing which couplers to activate, i.e., considering "snakes" of various lengths (cf. Fig. 2). This presents a unique opportunity for experimentally conducting finitesize scaling studies of the novel phase transition between the MBL and thermal phases, whose nature remains an active area of theoretical investigation.

Hamming distance. Finally, we present a diagnostic of spatiotemporal order that, while quite unusual from the point of view of many-body physics, is tailor made for devices like Sycamore. The "quantum supremacy" experiment [5] started with an initial bitstring, time evolved it under a random circuit, and then probed the output state by sampling its probability distribution over all bitstrings. We present a diagnostic for the different phases in our model that is in that vein, by considering the probability distribution of Hamming distances between the initial and time-evolved states.

Unlike ergodic dynamics, which quickly turns an initial bitstring state into a random state spread out over the entire computational basis, MBL prevents an initial state 
from veering too far from its initial condition. This fact can be quantified by the Hamming distance $d[80,81]$, which counts the minimum number of bit flips necessary to turn a bitstring $\sigma \in\{0,1\}^{L}$ into another, $\sigma^{\prime}$ : for example, $d=0$ $(L)$ only for identical (flipped) bitstrings, while typically $d=L / 2$ between two random bitstrings. Given a computational basis state $|\psi(0)\rangle=|\sigma\rangle$ and its time evolution after $n$ Floquet cycles, $|\psi(n)\rangle$, we can define the Hamming distance distribution

$$
P_{n}(d)=\left\langle\psi(n)\left|\Pi_{\sigma}(d)\right| \psi(n)\right\rangle,
$$

where $\Pi_{\sigma}(d)$ is the projector on bitstrings $\sigma^{\prime}$ that are a Hamming distance $d$ away from $\sigma$. We note that the average of the Hamming distance distribution, $\bar{d}=\sum_{d} P_{n}(d) d$, is information that can also be extracted from local expectation values of $Z$, since $2 \bar{d}=L-\sum_{i}(-1)^{\sigma_{i}}\left\langle Z_{i}(t)\right\rangle$; in particular, for the polarized initial state, this becomes a global observable, the total magnetization $\sum_{i} Z_{i}$. However, the full distribution $P(d)$ requires measuring the probabilities of entire bitstrings - a natural task for a programmable quantum simulator such as Sycamore that may instead be impractical or impossible on other platforms where such detailed readout is unavailable. While measuring the average $\bar{d}$ is enough to discriminate between MBL and ergodic phases, and a detailed measurement of the entire distribution $P_{n}(d)$ (particularly of its tails) would require considerably more sampling, it is nonetheless useful to have this capability. Even a coarse estimate of the distribution's width would be informative about the size of the subset of Hilbert space explored by the initial state during the dynamics, which in turn relates to the localization length (i.e., the spatial extent of the local integrals of motion).

Figures 4(d)-4(f) show data for the Hamming distance distribution $P_{n}(d)$ [Eq. (14)] in consecutive Floquet cycles at late times, $n_{1}=10^{4}$ and $n_{2}=n_{1}+1$, in the three phases. In the DTC phase [Fig. 4(d)], $P_{n}(d)$ remains peaked near $d=0$ (the initial bitstring) at even $n$ and, symmetrically, near $d=L$ (the globally flipped initial bitstring) at odd $n$. On the contrary, in the MBL paramagnet [Fig. 4(f)] $P_{n}(d)$ remains peaked near $d=0$ at all times. The behavior of both MBL phases should be contrasted with that of the thermal phase [Fig. 4(e)], where the Hamming distance distribution quickly becomes peaked at $d=L / 2$.

\section{EFFECT OF NOISE}

The discussion in the previous section shows that the Sycamore device has, in principle, all the ingredients necessary to stabilize and detect a DTC phase. We now address the important question of the robustness of the implementation and diagnostics to errors (in the form of noisy gates, environmental decoherence, and spurious time dependence of the circuit parameters). These give a signal that will be decaying in time, in practice. As discussed below, estimates of current noise thresholds predict that the distinctive temporal signatures of the DTC order should still be visible for multiple hundreds of driving periods. We emphasize again that spatial randomness is an inherent part of the DTC Floquet circuit, so small calibration errors between target gates and actual circuit elements are not a problem, provided these are reliably repeatable in time to give a Floquet circuit.

We model noise by considering one- and two-qubit depolarizing error models [82], acting on the system's density matrix $\rho$ as

$$
\begin{aligned}
& \Phi_{i}^{(1 q)}(\rho)=\left(1-p_{1}\right) \rho+\frac{p_{1}}{3} \sum_{\alpha \neq 0} \sigma_{\alpha, i} \rho \sigma_{\alpha, i}, \\
& \Phi_{i j}^{(2 q)}(\rho)=\left(1-p_{2}\right) \rho+\frac{p_{2}}{15} \sum_{\alpha, \beta}^{\prime} \sigma_{\alpha, i} \sigma_{\beta, j} \rho \sigma_{\alpha, i} \sigma_{\beta, j}
\end{aligned}
$$

[the primed sum denotes $(\alpha, \beta) \neq(0,0)$ ]. Each singlequbit gate acting on a qubit $i$ is followed by an application of the channel $\Phi_{i}^{(1 q)}$; each two-qubit gate on bond $(i, j)$ is followed by $\Phi_{i j}^{(2 q)}$. Conservative order-of-magnitude estimates for the depolarizing error rates with current technology $[5,67]$ are $p_{1} \approx 10^{-3}$ and $p_{2} \approx 10^{-2}$. The additional errors introduced by the active single-qubit rotations in the definition of $\widetilde{G}$ [Eq. (6)] can be taken into account approximately by enhancing the values of $p_{1}, p_{2}$. In the following we set $p_{2}=p, p_{1}=p / 10$, and refer to the single parameter $p$ as the "Pauli error rate" unless otherwise specified.

Channels (15) subsume the effect of fairly generic experimental errors, e.g., environmental decoherence, temporally random fluctuations of gate parameters, etc. In reality the errors may be anisotropic, e.g., $Z$ Pauli errors (phase flip) may be more or less frequent than $X$ (bitflip) errors. While this issue can be completely neglected in ergodic circuits [5], where each qubit's Bloch sphere is quickly scrambled and the error model is made effectively isotropic, in this MBL setting this need not be true. Indeed, in structured evolutions that explore their Hilbert space unevenly, the effect of errors depends on the details of the circuit. Nonetheless, in the absence of more detailed device-specific error modeling, the depolarizing model is a reasonable choice in that it involves all Pauli errors. We have additionally verified that our conclusions do not change qualitatively under a non-Pauli error model (the single-qubit amplitude-damping channel [82]); see Appendix B.

Quantum channels such as Eq. (15) can be "unraveled" into stochastic unitary evolutions $[83,84]$. Let us focus on the one-qubit channel $\Phi_{i}^{(1 q)}$ for simplicity. Its effect can be thought of as follows: after acting with each single-qubit gate $R_{i}^{x}$ from Eq. (7), the experimentalists toss a biased coin; with probability $p_{1}$, they apply an additional gate 
("error") drawn at random from $\left\{X_{i}, Y_{i}, Z_{i}\right\}$; otherwise, they apply $I$ (i.e., they do nothing). After $n$ cycles they get a pure state $\left|\psi_{\mathbf{r}}(n)\right\rangle$, where the label $\mathbf{r}$ keeps track of the error record, i.e., which error gates were applied, where and when during the entire evolution. Iterating this stochastic process gives an ensemble of pure-state unitary evolutions ("quantum trajectories" [85]) $\left\{\left|\psi_{\mathbf{r}}(n)\right\rangle\right\}$ that can be used to recover the density matrix $\rho(t)$ resulting from the real noisy evolution:

$$
\rho(n) \simeq \frac{1}{N_{\mathbf{r}}} \sum_{\mathbf{r}}\left|\psi_{\mathbf{r}}(n)\right\rangle\left\langle\psi_{\mathbf{r}}(n)\right|
$$

with $N_{\mathbf{r}}$ the number of sampled trajectories (this becomes exact in the limit $N_{\mathbf{r}} \rightarrow \infty$ ). Thus, at the expense of simulating multiple trajectories, one can evolve pure states instead of density matrices, greatly reducing the amount of memory needed for the computation.

Aside from their computational usefulness, quantum trajectories also offer a conceptually appealing view of the underlying error process. By unraveling a channel as outlined above, it is possible to think of the combined effect of all nonideal processes taking place in the experiment as "digital," with discrete errors taking place at specific locations in spacetime during the circuit dynamics. In the "quantum supremacy" experiment, Ref. [5], it was argued that a single such digital error could completely randomize the output state: only "error-free" circuit realizations could contribute to the signal being measured in that work; hence, its decay as $(1-p)^{L n} \approx e^{-p L n}$ (for $p \ll 1$ ). Therefore, the signal's lifetime gets worse with increasing system size, $n^{\star} \sim 1 /(p L)$. This argument however need not hold for MBL dynamics, where information propagates very slowly in space. It is plausible to expect in this case that a "digital" error at a given location will only affect observables in its vicinity, rather than completely randomize the output state.

This expectation is borne out by numerical simulations of quantum trajectories. Given the depolarizing error model of Eq. (15), the autocorrelator $C_{i i}(n)=\left\langle Z_{i}(0) Z_{i}(n)\right\rangle$ inevitably decays in time. Even under the ideal DTC circuit (with perfect $\pi$ pulse $\epsilon=0$ and no $\theta_{i j}$ couplings) one can see that $Z$ operators decay exponentially: $Z_{i}$ is invariant under the two-qubit gates but decays under the subsequent error, $\Phi_{i j}^{(2 q)}\left(Z_{i}\right)=\left(1-16 p_{2} / 15\right) Z_{i}$; after two iterations of this (with its two neighbors), $Z_{i}$ picks up a minus sign under the $\pi$ pulse, followed by the decay under single-qubit noise $\Phi_{i}^{(1 q)}\left(Z_{i}\right)=\left(1-4 p_{1} / 3\right) Z_{i}$. Thus, overall, $Z_{i} \mapsto-e^{-\gamma} Z_{i}$ over one Floquet cycle, with

$$
\gamma=-\ln \left[\left(1-\frac{16}{15} p_{2}\right)^{2}\left(1-\frac{4}{3} p_{1}\right)\right]
$$

an effective decoherence rate. Introducing nonideal elements to the DTC drive $\left(\epsilon \neq 0, \theta_{i j} \neq 0\right.$, etc.) is not going
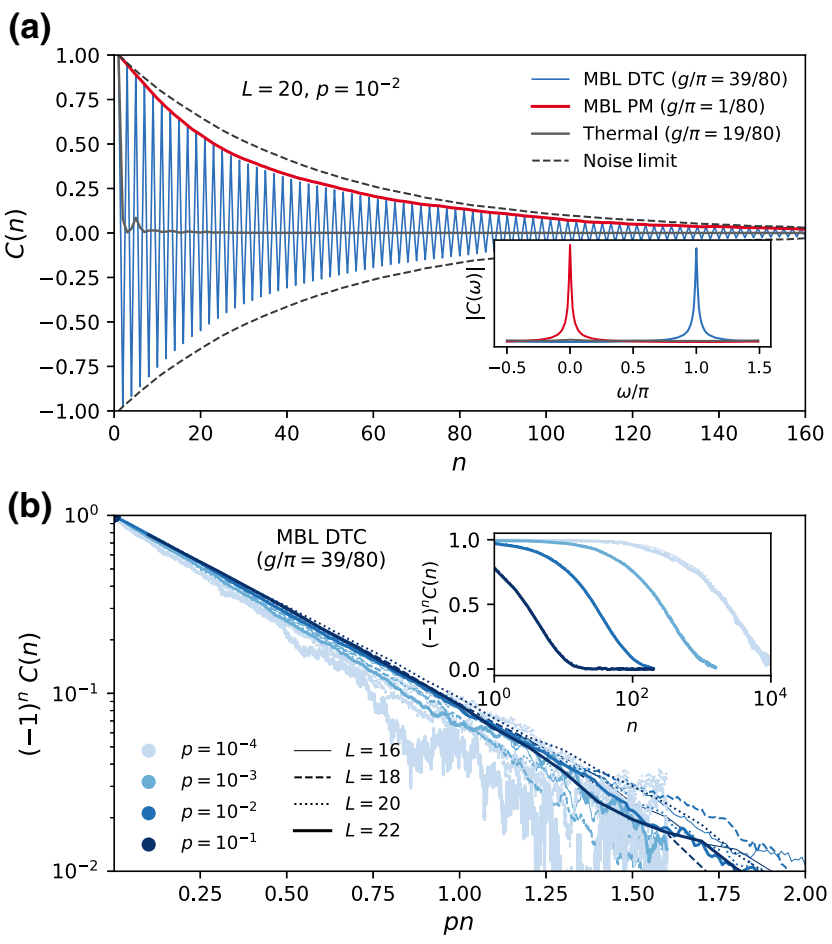

FIG. 6. Noisy dynamics. (a) Time evolution of the spatially averaged correlator $C(n)$ for a circuit with $L=20$ qubits in the presence of depolarizing noise $\left(p=10^{-2}\right)$ for the MBL DTC, MBL paramagnetic, and thermal phases, starting from a fixed bitstring state. The dashed line is the noise limit $e^{-\gamma t}$ (see the main text). Inset: Fourier transforms of the signals show (broadened) peaks at $\omega=\pi$ for the MBL DTC and $\omega=0$ for the MBL PM. (b) DTC signal for different system sizes $L$ and error rates $p$. All curves in the main panel overlap within statistical error, showing that the signal does not depend on $L$, and depends on $p$ only through the product $p n$, proportional to the number of accumulated errors per site. Inset: same data versus the number of Floquet cycles $n$. Different system sizes are indistinguishable.

to counter this decay; rather, it will generically include a (finite, transient) amount of "internal decoherence." The DTC signal is thus expected to be bounded by $\pm e^{-\gamma n}$. The data in Fig. 6(a) show a DTC signal with amplitude close to the maximal level allowed by noise.

Already with current hardware, this would yield a detectable DTC signal for hundreds of Floquet cycles. Indeed, the measurement task consists of resolving the expectation $\left\langle Z_{i}(n)\right\rangle$ (which is small, decaying as $e^{-\gamma n}$ at late times) of a binary variable with standard deviation $\sqrt{1-\left\langle Z_{i}(n)\right\rangle^{2}} \simeq 1$; this requires repeating the same experiment $N_{s} \gg 1$ times, which is not a problem for the Sycamore device given its high speed of operation (for the "quantum supremacy" experiment [5] $N_{s}=10^{6}$ samples were obtained in a few minutes). Equating the signal to the statistical noise floor then gives $e^{-\gamma n} \sim 1 / \sqrt{N_{s}}$; i.e., the signal can be resolved up to $n \leq n^{\star} \simeq(1 / 2 \gamma) \ln \left(N_{s}\right)$. Letting $p=10^{-2}$ (a conservative estimate for the present 
technology) and $N_{s}=10^{6}$ we obtain $n^{\star}=303$ Floquet cycles. We also note here that measurement is currently the lowest-fidelity process on Sycamore, with an average error rate of approximately $2.5 \%$; however, this process happens only once per run, and thus its effect does not scale with the size or depth of the circuit [86]. The estimate above would improve logarithmically with the number of samples $N_{s}$, but most importantly it would improve linearly in the inverse Pauli error, which is set to see substantial improvements in the future.

Finally, some remarks about the statistics of sampling the DTC signal. First, measurement is destructive - reading out the DTC signal at time $n$ arrests the evolution, which then needs to be started over for additional samples. Thus, producing an experimental version of Fig. 6(a) up to $n_{\max }$ cycles would require a number of runs scaling with $n_{\max }$. However, one could more economically extract robust evidence of spatiotemporal order from $O(1)$ time points, e.g., a snapshot at cycle $n$ (revealing spatial glassy order), one at $n+1$ (revealing the inversion of the glassy pattern), and one at $2 n$ (revealing the stability of the original glassy pattern). Second, because we are ultimately interested in the quantity $\overline{\left\langle Z_{i}(n)\right\rangle}$, averaged not only over quantum measurements but also over independent disorder realizations of the circuit for each starting state, the $N_{s}=N_{d} N_{q}$ experimental runs will be divided in practice between $N_{d}$ disorder realizations and $N_{q}$ separate runs of each circuit for quantum averaging. Benchmarking and calibrating a given realization of $U_{F}$ is more experimentally demanding than multiple runs of the same circuit; $N_{q} \sim O\left(10^{4}-10^{6}\right)$ and $N_{d} \sim O(10-100)$ seems feasible in the near term, which should provide enough averaging to resolve the signal.

In contrast to the long-lived temporal signal in the DTC phase, the signal from a circuit in the ergodic phase decays within a few Floquet cycles. In the thermal phase, the signal lifetime is not limited by external noise but rather by internal decoherence, i.e., by the system itself acting as a bath for the local observable [43], as shown in the error-free simulations of Fig. 4(b).

Because the signal's lifetime in the DTC is only limited by external sources of error, future hardware improvements would directly translate to potentially much longerlived realizations of the time crystal phase, as shown in Fig. 6(b). Furthermore, the signal's decay rate does not scale with size, suggesting that only errors in the vicinity of a given qubit cause damage to the local DTC signal. To confirm this picture, we have also simulated the dynamics of a system where $a$ single bond $(i, i+1)$ is subject to decoherence, and we find that the local DTC signal $C_{j j}(n)$ decays as $e^{-n / \tau_{j}}$ with a time constant that diverges exponentially in the spatial distance from the faulty bond, $\tau_{j} \sim$ $\exp |j-(i+1 / 2)| / \xi$; see Appendix C. Thus, when all bonds are noisy, by far the dominant source of decoherence for the signal at any site $j$ is the noise in its immediate vicinity, and the decay is to a very good approximation independent of $L$.

In sum, conservative estimates of noise levels suggest that Sycamore should already be able to observe a DTC signal for hundreds of Floquet cycles, which is on par with what was observed in FirstGen experiments, but with significant improvements expected as the hardware continues to advance. Importantly, the signal decay time does not directly scale with system size, so that the platform can be scaled up in size without a corresponding cost in experimental lifetime.

\section{DISCUSSION AND OUTLOOK}

\section{A. Summary}

In this work we have considered the question: what does the dawning age of NISQ devices and programmable quantum simulators have in store for quantum many-body physics? We have observed that, while these devices offer universal gate sets that can in principle simulate any quantum system, their limitation in coherence time practically favors certain simulation targets over others in the near term. Thus, when thinking of these devices as experimental platforms for many-body quantum mechanics, it is important to engage with their strengths and limitations, which are quite different from, and in some ways complementary to, those of the more traditional arenas for quantum many-body physics. This requires developing physical insight and intuition matching those needed in materials physics (regarding the choice of chemical compound, its synthesis, the selection and optimization of the experimental platform, and its theoretical modeling) or in cold-atomic systems (regarding the choice of atom or molecule, the cooling and loss suppression strategies, Hamiltonian engineering, and observable readout).

In the spirit of tailoring the application to what is most natural for the device in the near term, we noted that unitary circuits implement various kinds of driven quantum evolutions more straightforwardly than they do time-independent Hamiltonians. We have thus focused on out-of-equilibrium many-body phases in driven (Floquet) systems. Specifically, we have pointed to the Floquet discrete time crystal as a candidate well suited as a "physics-forward" simulation task on Sycamore; this phase is simultaneously interesting as the first example of an intrinsically nonequilibrium many-body phase of matter, a good fit for Sycamore's capabilities, and not yet realized in any other experimental platform. We have shown through detailed numerical simulations that the Floquet DTC can be stabilized on Sycamore over a range of realistic parameters, even under conservative assumptions about gate calibration error, and that all facets of the DTC spatiotemporal order can be compellingly revealed using the device's extensive capability for initialization and site-resolved readout. 
We have also addressed the effects of noise and decoherence on detecting the DTC spatiotemporal order. While all quantum simulators have to contend with the effects of environmental decoherence, the Sycamore platform has an edge insofar as the noise rates have been benchmarked with great care (while a full characterization of the noise processes is an ongoing research effort [87]). This would make it easier, in practice, to disentangle the effects of "internal" and "external" decoherence upon observing a decaying signal in time. Further, the great control afforded by this platform could also permit the use of various "echo sequences" (such as that used in the NMR experiment $[35,36])$ to further separate the effects of internal and external decoherence. We note that the former is a matter of principle: if even in an ideal, noise-free model the signal is eventually destroyed by internal decoherence (i.e., quantum thermalization), then the system does not realize a DTC phase (this is true of all FirstGen DTC experiments). On the other hand, if the signal's lifetime is limited by external decoherence (i.e., environmental noise and control errors), then this is an issue of engineering and, as such, will see sustained improvement with future hardware innovations.

Our proposal falls squarely in the latter category. The signal lifetime, already in the hundreds of cycles with current technology, is predicted to steadily increase with hardware improvements. The prospects for increasing the spatial size of the system are also promising. We have shown that the DTC order is sensitive to noise only locally, so that its lifetime is not negatively affected by increasing system size. The main constraint on the number of qubits thus becomes the geometry of the device.

\section{B. Directions for future research}

We conclude by mentioning interesting directions for future work along these lines. A set of mild variations of the setup proposed here can realize and probe a host of other interesting questions. Among these are prethermal time crystals [50], in particular in two dimensions. Experimental requirements are essentially identical to those we outlined, except of course for using all qubits and couplers on Sycamore's two-dimensional grid, rather than a onedimensional subset of them. Floquet symmetry-protected topological phases [88] are another natural target. These would require implementing a circuit that respects an Ising symmetry to a good approximation, and are thus a good target for future tests of high-precision many-body simulations. Among two-dimensional nonequilibrium phases, the "anomalous" (or "chiral") Floquet insulator [89-91] is another interesting target for simulation. This phase, where a MBL bulk coexists with quantized, chiral information flow at the edge, would also be realizable as a quantum circuit within Sycamore's gate set. Specifically, its circuit implementation would consist of five steps: four of them are given by near-SWAP gates (i.e., angles $\phi \simeq \pi, \theta \simeq \pi / 2$ in Sycamore's two-qubit gate set, with tolerance for sufficiently small imperfections), and the fifth is given by single-qubit disorder (e.g., $Z$ rotations by a site-dependent angle). The need for disorder only in single-qubit gates makes this particularly easy for Sycamore, as disorder realizations can be generated without additional calibration of two-qubit gates. Thus, the gate set poses no problem. What may require further technological progress is size: the chiral Floquet insulator, and its signature quantized transport of quantum information at the edge, requires a clear demarcation between bulk and boundary, with states on distinct edges not interacting with one another. This may be out of reach with the current approximately $(6 \times 8)$-sized device. A precise determination of requirements is a task for future research, as is the design of scalable protocols to measure the quantized flow of information within accessible coherence times.

Separately, quantum circuits are increasingly being studied as toy models for exploring a host of foundational questions in quantum statistical mechanics ranging from quantum chaos [92-99] to the dynamics of quantum entanglement $[95,100,101]$ to the emergence of hydrodynamics $[95,102]$. Exploring some of these issues experimentally could have a transformational impact on our understanding.

Finally, a direction we leave for future study is that of estimating the classical computing resources needed to simulate the proposed circuits. Circuits implemented on a specific hardware platform in the presence of finite errors require careful estimates of classical computational resources. In general, however, we note there are no efficient classical algorithms for exploring the entire phase diagram in Fig. 3. Indeed, the nature of MBL-to-thermal phase transition is still a largely open question, in no small part because of severe finite-size effects plaguing numerical explorations [103-110]. Experiments on ana$\log$ quantum simulator platforms have already investigated many interesting features of the MBL phase [111-118]; the increased flexibility of digital platforms such as Sycamore may, in addition, enable experimental finite-size scaling studies of the MBL-to-thermal phase transition [cf. Fig. 5(d)], potentially reaching much larger sizes than existing numerical studies, which could lend important insights to some of these open questions.

\section{ACKNOWLEDGMENTS}

We thank Rahul Nandkishore and Siddharth Parameswaran for discussions. This work is supported with funding from the Defense Advanced Research Projects Agency (DARPA) via the DRINQS program (support for M.I., and analysis of noise benchmarking and coherence times). The views, opinions, and/or findings 
expressed are those of the authors and should not be interpreted as representing the official views or policies of the Department of Defense or U.S. Government. M.I. was also funded in part by a Google Research Award: Quantum Hardware For Scientific Research In Physics and by the Gordon and Betty Moore Foundation's EPiQS Initiative through Grants No. GBMF4302 and No. GBMF8686. Computations were carried out on Stanford Research Computing Center's Sherlock cluster. The work is in part supported by the Deutsche Forschungsgemeinschaft through the Cluster of Excellence ct.qmat (EXC 2147, Project ID 390858490) (support for R. M.). This work was supported in part by the U.S. Department of Energy, Office of Science, Basic Energy Sciences, under Early Career Award No. DE-SC0021111 (support for V.K. and theoretical analysis of Floquet phases of quantum circuits). V.K. also acknowledges support from the Sloan Foundation through a Sloan Research Fellowship.

\section{APPENDIX A: NECESSITY OF ISING-EVEN DISORDER}

Here we explain why stabilizing a MBL DTC phase in model (1) requires having disorder in the Ising-even couplings $J_{i j} Z_{i} Z_{J}$, whereas disorder in the longitudinal fields $h_{i} Z_{i}$ is insufficient. Considering the case $\theta_{i j}=0$ for simplicity (small nonzero values do not qualitatively change the argument), the time evolution over two consecutive periods is given by

$$
U_{F}^{2}=P_{2 g} e^{-i H_{z}[\mathbf{J}, \mathbf{h}]} P_{2 g} e^{-i H_{z}[\mathbf{J}, \mathbf{h}]},
$$

where $H_{z}[\mathbf{J}, \mathbf{h}] \equiv \sum_{i} J_{i} Z_{i} Z_{i+1}+h_{i} Z_{i}$ and $P_{2 g} \equiv \prod_{i} R_{i}^{x}$ $(2 g)=\prod_{i} e^{-i g X_{i}}$ is the imperfect $\pi$ flip, with $2 g \equiv \pi-\epsilon$. By using the fact that $Z_{i}$ anticommutes with the Ising symmetry $P_{\pi}=\prod_{i} X_{i}$, we rewrite Eq. (A1) as

$$
\begin{aligned}
U_{F}^{2} & =P_{-\epsilon} e^{-i P_{\pi} H_{z}[\mathbf{J}, \mathbf{h}] P_{\pi}} P_{-\epsilon} e^{-i H_{z}[\mathbf{J}, \mathbf{h}]} \\
& =P_{-\epsilon} e^{-i H_{z}[\mathbf{J},-\mathbf{h}]} P_{-\epsilon} e^{-i H_{z}[\mathbf{J}, \mathbf{h}]} .
\end{aligned}
$$

The crux of the argument is the fact that the fields $h_{i}$ have opposite signs in the two consecutive actions of $e^{-i H_{z}}$ : to leading order in $\epsilon$, their effects cancel ("echo out"). To see this in more detail, we may write $U_{F}^{2}$ as

$$
\begin{aligned}
U_{F}^{2}= & P_{-2 \epsilon} e^{-i P_{\epsilon} H_{z}[\mathbf{J},-\mathbf{h}] P_{\epsilon}^{\dagger}} e^{-i H_{z}[\mathbf{J}, \mathbf{h}]} \\
= & P_{-2 \epsilon} e^{-i P_{\epsilon} H_{z}[\mathbf{J}, 0] P_{\epsilon}^{\dagger}} e^{-i P_{\epsilon} H_{z}[0,-\mathbf{h}] P_{\epsilon}^{\dagger}} \\
& \times e^{-i H_{z}[0, \mathbf{h}]} e^{-i H_{z}[\mathbf{J}, 0]},
\end{aligned}
$$

where we have decomposed $e^{-i H_{z}[\mathbf{J}, \mathbf{h}]}$ into the (commuting) factors $e^{-i H_{z}[\mathbf{J}, 0]} e^{-i H_{z}[0, \mathbf{h}]}$. Now if we take the $\mathbf{J}$ couplings to be clean, $J_{i} \equiv J$, the above expression can be rewritten by isolating the disordered part as

$$
U_{F}^{2}=U_{\text {clean }}^{(1)} \cdot \prod_{i} e^{i h_{i} P_{\epsilon} Z_{i} P_{\epsilon}^{\dagger}} e^{-i h_{i} Z_{i}} \cdot U_{\text {clean }}^{(2)}
$$

Straightforward algebra yields

$$
e^{i h_{i} P_{\epsilon} Z_{i} P_{\epsilon}^{\dagger}} e^{-i h_{i} Z_{i}}=e^{-i \tilde{h}_{i} \hat{\mathbf{n}}_{i} \cdot \sigma_{i}},
$$

where $\hat{\mathbf{n}}_{i}$ is a unit vector and $\tilde{h}_{i}$ obeys

$$
\cos \tilde{h}_{i}=1-\sin ^{2}\left(h_{i}\right)(1-\cos \epsilon) ;
$$

hence, when $\epsilon \ll 1$, we have $\tilde{h}_{i} \approx \epsilon \sin h_{i} \ll 1$. Thus, the effective disorder strength in the fields $h_{i}$ is greatly reduced precisely in the regime where the DTC order should be found (small $\epsilon$ ), posing a problem for the stabilization of the MBL DTC. Note that this is not a problem at small $g$ $(\epsilon \approx \pi)$, where disorder in the onsite fields does not get echoed out and can stabilize a MBL paramagnet. Numerical simulations of the model confirm this scenario, giving only a MBL paramagnetic phase (at sufficiently small $g$ ) and an ergodic phase in the rest of parameter space.

To illustrate this, we have performed dynamics simulations of the model realizable in Sycamore, Eq. (7), with maximal disorder in the $h_{a / b / c}^{i j}$ angles (sampled uniformly from $[0,2 \pi]$ ), both with and without disorder in the $\phi$ angles (again the identification between controlledphase angles and Ising couplings is $\phi=4 J$ ). We use the same discrete-disorder model as in the main text, with $M=8$ values, $\bar{\phi}=\pi$, and disorder strength $W$ set to either $W=\pi / 2$ (as in the main text) or $W=0$. Finally, we take $\bar{\theta}=0$ and $\Delta \theta=\pi / 50$. The results are shown in Fig. 7 . While the MBL PM phase $(g=\pi / 80)$ is fully stabilized by the $h$ fields, with negligible effect of $W$, the MBL DTC ( $g=39 \pi / 80$, i.e., $\epsilon=\pi / 40)$ requires $W \neq 0$. In sum, in

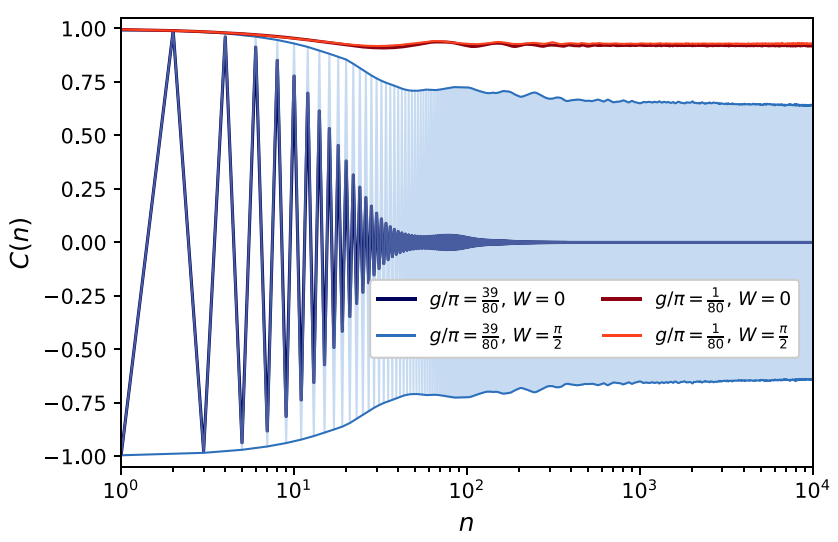

FIG. 7. Temporal autocorrelator $C(n)$ for $L=16$ qubits, averaged over position, 100 disorder realizations, and 100 initial states, with maximal disorder in the Ising-odd $h$ fields $(h \in$ $[0,2 \pi])$, and with disorder $W$ in the Ising-even $\phi$ angles. 


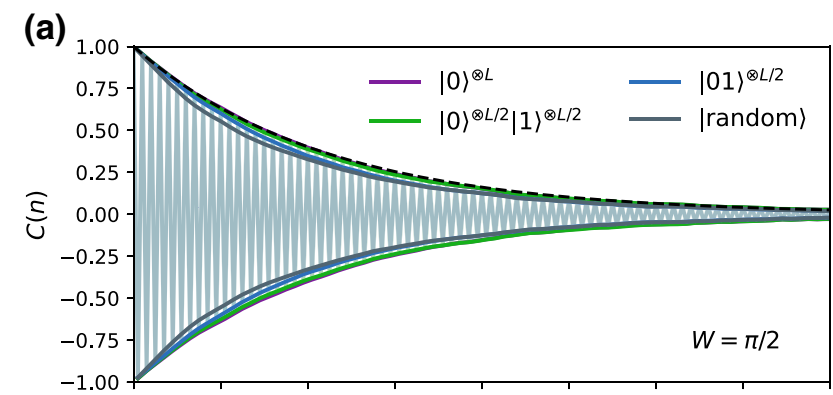

(b)

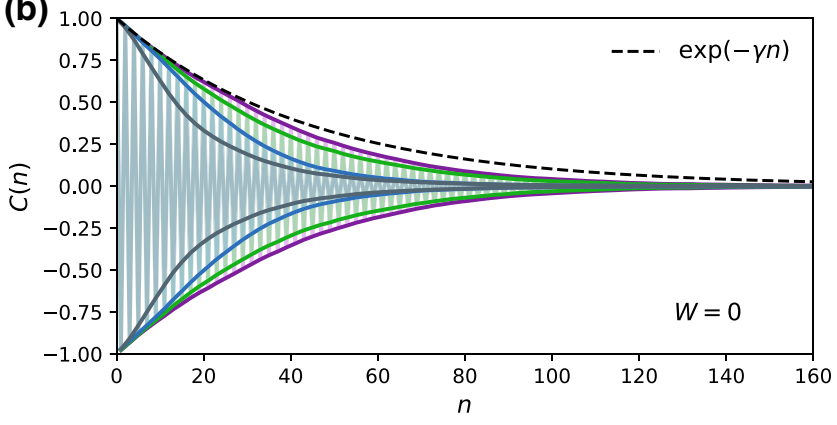

FIG. 8. (a) Temporal autocorrelator $C(n)$ for $L=20$ qubits in the presence of noise (rate $p=10^{-2}$ ) in the MBL DTC phase $(g / \pi=39 / 80$, disorder in the controlled-phase angles $W=$ $\pi / 2$ ) for the four initial bitstring states indicated in the legend. For each state, the data are averaged over position and at least $10^{3}$ combined realizations of disorder and noise (i.e., quantum trajectories). The dashed line indicates the decoherence bound $e^{-\gamma n}$, also present in Fig. 6(a), with $\gamma$ defined in Eq. (17). The bitstring indicated by |random $\rangle$ is |00101010100110111001〉. (b) Same simulation but without disorder in the controlled-phase angles, $W=0$ [with $\bar{\phi}=\pi$ as in (a)]. Other model parameters are $\Delta \theta=\bar{\theta}=\Delta h=\pi / 50$.

the absence of disorder in Ising-even interactions, disorder in the Ising-odd longitudinal fields $h_{i}$ is insufficient to stabilize the DTC phase.

Finally, to show that this phenomenon would be visible even in the presence of external decoherence, we have repeated the analysis in the presence of an error rate $p=10^{-2}$ (same as in Fig. 6). The results are displayed in Fig. 8. For $W=\pi / 2$, we have a genuine MBL DTC phase, showing very limited state-to-state variation and temporal autocorrelators $C(n)$ consistent with an $O(1)$ asymptotic value modulated by external decoherence, in line with the exponential envelope in Eq. (17). On the contrary, in Fig. 8(b) we turn off the disorder in the $\phi$ angles, setting $W=0$, and observe much stronger state-tostate fluctuations. Special "low temperature" states (such as the polarized one, or the one with only two domain walls) nearly saturate the decoherence envelope at early times; however, they start to decay more quickly after a few tens of cycles - at that point, one expects several bit flips have taken place due to the noise, and the states are progressively less "special." Typical ("high-temperature") bitstrings, on the other hand, immediately decay faster than decoherence alone would dictate, indicating an intrinsic instability. This distinctive behavior is evident well within the coherence time of approximately 100 cycles.

\section{APPENDIX B: EFFECT OF DIFFERENT NOISE MODELS}

\section{Control errors and decoherence}

In this work we have modeled the effects of noise and decoherence via a depolarizing channel. This is a justified assumption if the underlying dynamics is strongly scrambling, but not if it is highly structured, as in a MBL phase. It is thus important to study the effects of different noise models.

To simplify the comparison of different models, we restrict to single-qubit decoherence channels, $\Phi^{(1 q)}$. These are assumed to act after all gates, whether one or two qubits; so, e.g., an application of $R_{i}^{z}$ is followed by the action of $\Phi_{i}^{(1 q)}$, while an application of $G_{i, j}$ is followed by the action of $\Phi_{i}^{(1 q)} \otimes \Phi_{j}^{(1 q)}$. We consider four families of quantum channels [82], all parameterized by a rate $p \in[0,1]$ as follows.

1. Depolarizing channel,

$$
\Phi_{i}(\rho)=(1-p) \rho+\frac{p}{3}\left(X_{i} \rho X_{i}+Y_{i} \rho Y_{i}+Z_{i} \rho Z_{i}\right) .
$$

2. Bit-flip channel,

$$
\Phi_{i}(\rho)=(1-p) \rho+p X_{i} \rho X_{i} .
$$

3. Phase-flip channel,

$$
\Phi_{i}(\rho)=(1-p) \rho+p Z_{i} \rho Z_{i} .
$$

4. Amplitude-damping channel,

$$
\Phi_{i}(\rho)=A_{i} \rho A_{i}^{\dagger}+B_{i} \rho B_{i}^{\dagger}
$$

with

$$
A=\left(\begin{array}{cc}
1 & 0 \\
0 & \sqrt{1-p}
\end{array}\right), \quad B=\left(\begin{array}{cc}
0 & \sqrt{p} \\
0 & 0
\end{array}\right) .
$$

Unlike the depolarizing model, the other three act anisotropically on the Bloch sphere. Moreover, the amplitude-damping channel is not unital, i.e., does not preserve the maximally mixed state (it has $|0\rangle\langle 0|$ as its only fixed point).

The results of simulations of the dynamics under these error models, using the same method described in the main text, are shown in Fig. 9. We find that the depolarizing, bitflip, and amplitude-damping channels have similar effects, causing an exponential decay with a rate close to $p$, within 


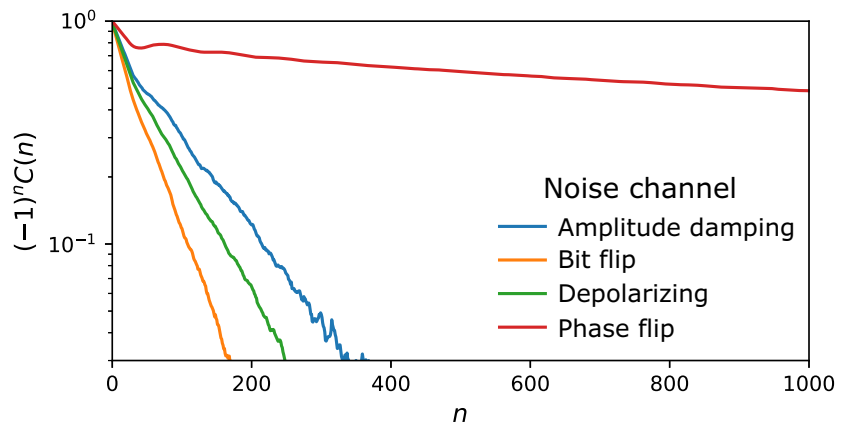

FIG. 9. Effect of different noise models on the DTC signal [autocorrelator $C(n)$, averaged over position and $10^{3}$ disorder realizations] for $L=20$ qubits at $g / \pi=39 / 80$. All noise channels are single qubit and have rate $p=3 \times 10^{-3}$ (see the text).

factors of order unity. On the contrary, the phase-flip error model causes a much slower decay (by over an order of magnitude in this case).

This behavior follows from the existence of local integrals of motion (lbits) in the MBL phase, which are nearly aligned with the $Z$ axis in these models. To the extent that the quantum jump operators $Z_{i}$ commute with the lbits, one can decouple the ideal dynamics (the Floquet unitary $U_{F}$ ) from the decoherence,

$$
\rho \mapsto U_{F}^{n}\left[\bigotimes_{i} \Phi_{i}^{n}(\rho)\right]\left(U_{F}^{\dagger}\right)^{n}
$$

If the initial state is itself an eigenstate of the $Z_{i}$, it is immune to the phase-flip decoherence and one recovers the ideal dynamics. Thus, the effect of decoherence is suppressed by how closely aligned the lbits are with the $Z$ axis. As the tilt is induced by the "transverse field" (pulse imperfection), one expects the rate of decoherence in this case to scale as $|g|$ in the MBL PM phase and as $|g-\pi / 2|$ in the MBL DTC. For the case of Fig. 9, this yields a lifetime enhancement of approximately $40 / \pi \simeq 13$, which is in line with the data.

Finally, we note that the depolarizing noise is effectively a weighted average of bit-flip and phase-flip noise, and is thus intermediate between the two. The amplitudedamping noise behaves similarly as well.

\section{Measurement error}

Here we consider the effect of (possibly correlated) readout errors on the various diagnostics studied in this paper. We model the measurement error as a stochastic process where the outcome of a qubit state measurement (in the $Z$ computational basis) is randomly flipped, with probability $p_{m}$, away from its correct value. A realistic estimate for $p_{m}$ on Sycamore is $p_{m} \simeq 2.5 \%$.

We start by considering local observables, such as $C_{i i}(n)=\left\langle Z_{i}(n) Z_{i}(0)\right\rangle$. Assuming that the initial state is a bitstring $|\psi\rangle=|\mathbf{s}\rangle \quad\left(s_{i} \in \mathbb{Z}_{2}\right)$ prepared perfectly, we have $C_{i i}(n)=s_{i}\left\langle Z_{i}(n)\right\rangle$; letting $A_{ \pm}=\left\langle\left[1 \pm Z_{i}(n)\right] / 2\right\rangle$ be the probability that qubit $i$ points up (down) at time $n$, the result of the noisy measurement process is $Z_{i}=+1$ with probability $A_{+}\left(1-p_{m}\right)+A_{-} p_{m}$, and $Z_{i}=-1$ with probability $A_{+} p_{m}+A_{-}\left(1-p_{m}\right)$. In all, the estimate for $C_{i i}(n)$ with measurement error, $C_{i i}^{\mathrm{ME}}(n)$, becomes

$$
\begin{aligned}
C_{i i}^{\mathrm{ME}}(n) & =A_{+}\left(1-2 p_{m}\right)-A_{-}\left(1-2 p_{m}\right) \\
& =\left(1-2 p_{m}\right) C_{i i}(n)
\end{aligned}
$$

where we used the fact that $C_{i i}(n)=A_{+}-A_{-}$. This argument goes through for each site $i$, independent of any correlations in the measurement errors; hence, averaging over position yields $C^{\mathrm{ME}}=\left(1-2 p_{m}\right) C(n)$, i.e., a damping by a time-independent overall prefactor. This lowers the signal's lifetime by a modest amount, but does not qualitatively change its behavior.

Correlations in readout errors have an effect on quantities that specifically diagnose the spatial glassiness, such as the Edwards-Anderson (spin glass) order parameter, Eq. (13). Qubits on Sycamore are measured in groups of six via a frequency-multiplexing scheme [5], which could introduce correlations in the measurement errors. As we envision an effective one-dimensional system living on a path that zigzags through Sycamore, such correlations in measurement errors will in general be nonlocal in the one-dimensional system. We consider an extreme scenario where the system's qubits are partitioned into groups $G_{1}, G_{2}, \ldots$ and the measurement errors are perfectly correlated within each group (and uncorrelated between groups): for each set $G_{\alpha}$, with probability $p_{m}$, all qubits in $G_{\alpha}$ are measured incorrectly (i.e., flipped); otherwise, they are all measured correctly. (One could study models with imperfect correlations in measurement errors and arrive at similar conclusions.) The EdwardsAnderson order parameter is measured by first obtaining a quantum average of $s_{i j} \equiv\left\langle Z_{i}(n) Z_{j}(n)\right\rangle$, then computing $\chi^{\mathrm{SG}}=(1 / L) \sum_{i, j} s_{i j}^{2}$, and averaging the result over disorder realizations. For two qubits in the same group $G_{\alpha}, s_{i j}$ does not suffer any measurement error - either both qubits flip, or neither does, leaving the product fixed. However, for two qubits in distinct groups, we have $s_{i j} \mapsto s_{i j}$ [(1$\left.\left.p_{m}\right)^{2}+p_{m}^{2}-2 p_{m}\left(1-p_{m}\right)\right]=s_{i j}\left(1-2 p_{m}\right)^{2}$. Adding up all contributions, we find that

$$
\chi^{\mathrm{SG}, \mathrm{ME}}=\chi_{\mathrm{diag}}^{\mathrm{SG}}+\left(1-2 p_{m}\right)^{4}\left[\chi^{\mathrm{SG}}-\chi_{\mathrm{diag}}^{\mathrm{SG}}\right],
$$

where we introduced the "diagonal" sum

$$
\chi_{\mathrm{diag}}^{\mathrm{SG}}=\frac{1}{L} \sum_{\alpha} \sum_{i, j \in G_{\alpha}} s_{i j}^{2} .
$$

The case of uncorrelated measurement errors is recovered by setting $G_{\alpha} \equiv\left\{i_{\alpha}\right\}$ (each qubit forms its own set), which 
gives $\chi_{\text {diag }}^{\mathrm{SG}}=1$ and, thus,

$$
\chi^{\mathrm{SG}, \mathrm{ME}}=1+\left(1-2 p_{m}\right)^{4}\left(\chi^{\mathrm{SG}}-1\right) .
$$

Because all partial sums are positive, one has $\chi_{\text {diag }}^{\mathrm{SG}} \geq 1$ in the presence of correlations. It follows, by comparing Eqs. (B2) and (B3), that correlations in measurement errors in fact slightly enhance the lifetime of $\chi^{\mathrm{SG}}$ (compared to uncorrelated errors), and in any case do not qualitatively change its behavior.

\section{APPENDIX C: LOCALITY OF DECOHERENCE IN MBL PHASES}

Here we discuss the effects of MBL on the propagation of decoherence in the system. In a strongly ergodic system, an error anywhere in the system quickly randomizes the entire wavefunction (within a ballistic lightcone). In a MBL system, on the other hand, an error at a given location has effects only within a logarithmic "lightcone" $[119,120]$, so, for all practical purposes, the effects of decoherence are local-loss of coherence at a given site is dominantly the consequence of errors at that site or in its immediate vicinity.

We illustrate this point by simulating a qubit chain where the evolution is ideal and unitary everywhere, except for a single location in space. For specificity, we choose a bond (qubits $i=0,1$ ); there, the same (one- and two-qubit) depolarizing noise model used in the main text acts at every time step. We then measure the DTC signal, i.e., the staggered autocorrelator $(-1)^{n} C_{i i}(n)$ at all qubits $i$.

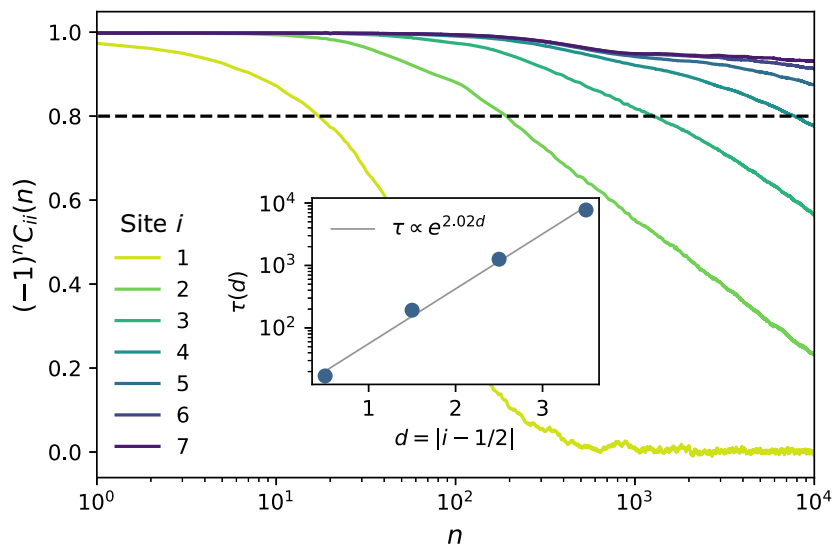

FIG. 10. Numerical simulation of $L=16$ qubits in the DTC phase with depolarizing noise (as in Fig. 6, with $p=0.01$ ) acting only on a single bond (qubits $i=0,1$; boundary conditions are periodic), averaged over $10^{4}$ realizations of disorder. The dashed line represents an arbitrary threshold (0.8) used to extract a "decay time" $\tau$ for each site. Inset: DTC signal's decay time, $\tau(d)$, diverges exponentially in the distance $d$ from the noisy bond, consistent with the presence of exponentially localized integrals of motion in the MBL DTC phase.
The results are shown in Fig. 10. We find that the decay time scale for qubit $i$ diverges as a function of its distance $d$ from the faulty bond as $\tau(d) \sim e^{d / \xi}$. This is consistent with the expectation for a system with exponentially localized lbits: each lbit is depolarized at a rate proportional to its overlap with the noisy sites, which in turn is set by the exponentially decaying envelope of the lbit, $\tau_{i}^{z} \sim \sum_{j} e^{-|i-j| / \xi} O_{j}$, where each $O_{j}$ is supported around site $j$.

As a consequence of this, loss of the DTC signal at a given position is chiefly the result of errors at or near that position, even after a long time. Thus, the overall lifetime of the DTC signal is approximately independent of system size, as seen in Fig. 6.

[1] I. M. Georgescu, S. Ashhab, and F. Nori, Quantum simulation, Rev. Mod. Phys. 86, 153 (2014).

[2] E. Altman et al., Quantum Simulators: Architectures and Opportunities, PRX Quantum 2, 017003 (2019).

[3] J. Preskill, Quantum computing in the NISQ era and beyond, Quantum 2, 79 (2018).

[4] Much of nature routinely carries out processes that are not simulable on a classical computer, but these are not recognizably computational tasks on highly controllable and thus recognizably computational devices. See Ref. [121] for a discussion of this point.

[5] F. Arute et al., Quantum supremacy using a programmable superconducting processor, Nature 574, 505 (2019).

[6] S. Aaronson, Certified randomness from quantum supremacy, http://pirsa.org/displayFlash.php?id=1807 0057 (2018), online; accessed: 2020-07-03.

[7] F. Arute et al., Hartree-fock on a superconducting qubit quantum computer, Science 369, 1084 (2020).

[8] F. Arute et al., Quantum approximate optimization of non-planar graph problems on a planar superconducting processor, Nat. Phys. 17, 332 (2021).

[9] D. P. DiVincenzo, The physical implementation of quantum computation, Fortschritte der Physik 48, 771 (2000).

[10] I. Bloch, J. Dalibard, and W. Zwerger, Many-body physics with ultracold gases, Rev. Mod. Phys. 80, 885 (2008).

[11] C. Gross and I. Bloch, Quantum simulations with ultracold atoms in optical lattices, Science 357, 995 (2017).

[12] R. P. Feynman, Simulating physics with computers, Int. J. Theor. Phys. 21, 467 (1982).

[13] S. Lloyd, Universal quantum simulators, Science 273, 1073 (1996).

[14] D. Wecker, M. B. Hastings, N. Wiebe, B. K. Clark, C. Nayak, and M. Troyer, Solving strongly correlated electron models on a quantum computer, Phys. Rev. A 92, 062318 (2015).

[15] S. McArdle, S. Endo, A. Aspuru-Guzik, S. C. Benjamin, and X. Yuan, Quantum computational chemistry, Rev. Mod. Phys. 92, 015003 (2020).

[16] A. Rahmani, K. J. Sung, H. Putterman, P. Roushan, P. Ghaemi, and Z. Jiang, Creating and Manipulating a Laughlin-Type $v=1 / 3$ Fractional Quantum Hall State on 
a Quantum Computer with Linear Depth Circuits, PRX Quantum 1, 020309 (2020).

[17] F. Harper, R. Roy, M. S. Rudner, and S. Sondhi, Topology and broken symmetry in floquet systems, Annu. Rev. Condens. Matter Phys. 11, 345 (2020).

[18] D. A. Huse, R. Nandkishore, V. Oganesyan, A. Pal, and S. L. Sondhi, Localization-protected quantum order, Phys. Rev. B 88, 014206 (2013).

[19] V. Khemani, A. Lazarides, R. Moessner, and S. Sondhi, Phase Structure of Driven Quantum Systems, Phys. Rev. Lett. 116, 250401 (2016).

[20] D. V. Else, B. Bauer, and C. Nayak, Floquet Time Crystals, Phys. Rev. Lett. 117, 090402 (2016).

[21] C. W. von Keyserlingk, V. Khemani, and S. L. Sondhi, Absolute stability and spatiotemporal long-range order in floquet systems, Phys. Rev. B 94, 085112 (2016).

[22] V. Khemani, R. Moessner, and S. Sondhi, A brief history of time crystals, arXiv:1910.10745 (2019).

[23] K. Sacha and J. Zakrzewski, Time crystals: A review, Rep. Prog. Phys. 81, 016401 (2018).

[24] M. Faraday, On a peculiar class of acoustical figures; and on certain forms assumed by groups of particles upon vibrating elastic surfaces, Philos. Trans. R. Soc. London 121, 299 (1831).

[25] R. E. Goldstein, Coffee stains, cell receptors, and time crystals: Lessons from the old literature, Phys. Today 71, 32 (2018).

[26] M. Holthaus and M. E. Flatte, Subharmonic generation in quantum systems, Phys. Lett. A 187, 151 (1994).

[27] K. Sacha, Modeling spontaneous breaking of timetranslation symmetry, Phys. Rev. A 91, 033617 (2015).

[28] A. Russomanno, F. Iemini, M. Dalmonte, and R. Fazio, Floquet time crystal in the lipkin-meshkov-glick model, Phys. Rev. B 95, 214307 (2017).

[29] S. Pal, N. Nishad, T. S. Mahesh, and G. J. Sreejith, Temporal Order in Periodically Driven Spins in Star-Shaped Clusters, Phys. Rev. Lett. 120, 180602 (2018).

[30] T. L. Heugel, M. Oscity, A. Eichler, O. Zilberberg, and R. Chitra, Classical Many-Body Time Crystals, Phys. Rev. Lett. 123, 124301 (2019).

[31] V. K. Kozin and O. Kyriienko, Quantum Time Crystals from Hamiltonians with Long-Range Interactions, Phys. Rev. Lett. 123, 210602 (2019).

[32] N. Y. Yao, C. Nayak, L. Balents, and M. P. Zaletel, Classical discrete time crystals, Nat. Phys. 16, 438 (2020).

[33] S. Choi, J. Choi, R. Landig, G. Kucsko, H. Zhou, J. Isoya, F. Jelezko, S. Onoda, H. Sumiya, V. Khemani, C. von Keyserlingk, N. Y. Yao, E. Demler, and M. D. Lukin, Observation of discrete time-crystalline order in a disordered dipolar many-body system, Nature 543, 221 (2017).

[34] J. Zhang, P. W. Hess, A. Kyprianidis, P. Becker, A. Lee, J. Smith, G. Pagano, I.-D. Potirniche, A. C. Potter, A. Vishwanath, N. Y. Yao, and C. Monroe, Observation of a discrete time crystal, Nature 543, 217 (2017).

[35] J. Rovny, R. L. Blum, and S. E. Barrett, Observation of Discrete-Time-Crystal Signatures in an Ordered Dipolar Many-Body System, Phys. Rev. Lett. 120, 180603 (2018).

[36] J. Rovny, R. L. Blum, and S. E. Barrett, ${ }^{31} \mathrm{P}$ nmr study of discrete time-crystalline signatures in an ordered crystal of ammonium dihydrogen phosphate, Phys. Rev. B 97, 184301 (2018).

[37] H. Watanabe, M. Oshikawa, and T. Koma, Proof of the absence of long-range temporal orders in gibbs states, J. Stat. Phys. 178, 926 (2020).

[38] L. Kühn, über ein neues radiotelephonisches system, Elektrotechnische Zeitschrift 35, 816 (1914).

[39] P. Ponte, A. Chandran, Z. Papić, and D. A. Abanin, Periodically driven ergodic and many-body localized quantum systems, Ann. Phys. (N. Y) 353, 196 (2015).

[40] A. Lazarides, A. Das, and R. Moessner, Equilibrium states of generic quantum systems subject to periodic driving, Phys. Rev. E 90, 012110 (2014).

[41] D. M. Basko, I. L. Aleiner, and B. L. Altshuler, Metalinsulator transition in a weakly interacting many-electron system with localized single-particle states, Ann. Phys. (Amsterdam) 321, 1126 (2006).

[42] J. Z. Imbrie, On many-body localization for quantum spin chains, J. Stat. Phys. 163, 998 (2016).

[43] R. Nandkishore and D. A. Huse, Many-body localization and thermalization in quantum statistical mechanics, Annu. Rev. Condens. Matter Phys. 6, 15 (2015).

[44] D. A. Abanin, E. Altman, I. Bloch, and M. Serbyn, Colloquium: Many-body localization, thermalization, and entanglement, Rev. Mod. Phys. 91, 021001 (2019).

[45] A. Lazarides, A. Das, and R. Moessner, Fate of ManyBody Localization Under Periodic Driving, Phys. Rev. Lett. 115, 030402 (2015).

[46] P. Ponte, Z. Papić, F. Huveneers, and D. A. Abanin, ManyBody Localization in Periodically Driven Systems, Phys. Rev. Lett. 114, 140401 (2015).

[47] R. Moessner and S. L. Sondhi, Equilibration and order in quantum floquet matter, Nat. Phys. 13, 424 (2017).

[48] T. Mori, T. Kuwahara, and K. Saito, Rigorous Bound on Energy Absorption and Generic Relaxation in Periodically Driven Quantum Systems, Phys. Rev. Lett. 116, 120401 (2016).

[49] D. Abanin, W. De Roeck, W. Ho, and F. Huveneers, A rigorous theory of many-body prethermalization for periodically driven and closed quantum systems, Commun. Math. Phys. 354, 809 (2017).

[50] D. Else, B. Bauer, and C. Nayak, Prethermal Phases of Matter Protected by Time-Translation Symmetry, Phys. Rev. X 7, 011026 (2017).

[51] A. Rubio-Abadal, M. Ippoliti, S. Hollerith, D. Wei, J. Rui, S. L. Sondhi, V. Khemani, C. Gross, and I. Bloch, Floquet Prethermalization in a Bose-Hubbard System, Phys. Rev. X 10, 021044 (2020).

[52] P. Peng, C. Yin, X. Huang, C. r. Ramanathan, and P. Cappellaro, Observation of Floquet prethermalization in dipolar spin chains, Nat. Phys. 17, 444 (2021).

[53] W. W. Ho, S. Choi, M. D. Lukin, and D. A. Abanin, Critical Time Crystals in Dipolar Systems, Phys. Rev. Lett. 119, 010602 (2017).

[54] D. J. Luitz, R. Moessner, S. L. Sondhi, and V. Khemani, Prethermalization Without Temperature, Phys. Rev. X 10, 021046 (2020).

[55] H. Keßler, J. G. Cosme, C. Georges, L. Mathey, and A. Hemmerich, From a continuous to a discrete time crystal 
in a dissipative atom-cavity system, New J. Phys. 22, 085002 (2020).

[56] Y. Zhang, J. Gosner, S. M. Girvin, J. Ankerhold, and M. I. Dykman, Time-translation-symmetry breaking in a driven oscillator: From the quantum coherent to the incoherent regime, Phys. Rev. A 96, 052124 (2017).

[57] L. Fleishman and P. W. Anderson, Interactions and the anderson transition, Phys. Rev. B 21, 2366 (1980).

[58] A. L. Burin, Localization in a random xy model with long-range interactions: Intermediate case between singleparticle and many-body problems, Phys. Rev. B 92, 104428 (2015).

[59] N. Y. Yao, C. R. Laumann, S. Gopalakrishnan, M. Knap, M. Müller, E. A. Demler, and M. D. Lukin, Many-Body Localization in Dipolar Systems, Phys. Rev. Lett. 113, 243002 (2014).

[60] Note that, for the purpose of this article, we are not concerning ourselves with the open question of possible nonperturbative instabilities of MBL that may asymptotically destabilize localization in dimensions greater than one, or with power-law decaying interactions with any power [65]. These effects, if they exist, will happen for system sizes and time scales that are well beyond the capabilities of any near-term simulators.

[61] We thank S. Gopalakrishnan for a discussion of this point.

[62] G.-D. Lin, C. Monroe, and L.-M. Duan, Sharp Phase Transitions in a Small Frustrated Network of Trapped ion Spins, Phys. Rev. Lett. 106, 230402 (2011).

[63] S. Korenblit, D. Kafri, W. C. Campbell, R. Islam, E. E. Edwards, Z.-X. Gong, G.-D. Lin, L.-M. Duan, J. Kim, K. Kim, and C. Monroe, Quantum simulation of spin models on an arbitrary lattice with trapped ions, New J. Phys. 14, 095024 (2012).

[64] J. Zeiher, R. van Bijnen, P. Schauß, S. Hild, J.-y. Choi, T. Pohl, I. Bloch, and C. Gross, Many-body interferometry of a rydberg-dressed spin lattice, Nat. Phys. 12, 1095 (2016).

[65] W. De Roeck and F. m. c. Huveneers, Stability and instability towards delocalization in many-body localization systems, Phys. Rev. B 95, 155129 (2017).

[66] D. Aharonov and M. Ben-Or, in Proceedings of the Twenty-Ninth Annual ACM Symposium on Theory of Computing, STOC '97 (Association for Computing Machinery, New York, NY, USA, 1997), p. 176.

[67] R. Barends, C. Quintana, A. Petukhov, Y. Chen, D. Kafri, K. Kechedzhi, R. Collins, O. Naaman, S. Boixo, and F. Arute et al., Diabatic Gates for Frequency-Tunable Superconducting Qubits, Phys. Rev. Lett. 123, 210501 (2019).

[68] B. Foxen et al., Demonstrating a Continuous Set of TwoQubit Gates for Near-Term Quantum Algorithms, Phys. Rev. Lett. 125, 120504 (2020).

[69] There are only three independent angles because $Z_{1}+Z_{2}$ commutes with FS.

[70] I. D. Kivlichan, J. McClean, N. Wiebe, C. Gidney, A. Aspuru-Guzik, G. K.-L. Chan, and R. Babbush, Quantum Simulation of Electronic Structure with Linear Depth and Connectivity, Phys. Rev. Lett. 120, 110501 (2018).

[71] Any temporally random fluctuations and/or additional decoherence due to the execution of the active $Z$ rotations can be accounted for by increasing an effective "Pauli error rate"; we return to them when we discuss the noise model in Sec. IV.

[72] Localization is expected to be stable even with discrete (rather than continuous) disorder, provided the number of values $M$ in the discrete set is large enough $(M=2$ is pathological) [122]. Nonetheless, we note that discrete disorder falls outside the set of conditions required for a rigorous nonperturbative proof of MBL [42], and may thus generate resonances that eventually destabilize localization. However, any such effects would appear on a parametrically long time scale, akin to concerns regarding the stability of MBL in higher dimensions or with powerlaw interactions of any power [65]. These open issues are beyond the purview of this work, and will be invisible at the system sizes and times accessible to near-term devices.

[73] D. Pekker, G. Refael, E. Altman, E. Demler, and V. Oganesyan, Hilbert-Glass Transition: New Universality of Temperature-Tuned Many-Body Dynamical Quantum Criticality, Phys. Rev. X 4, 011052 (2014).

[74] B. Bauer and C. Nayak, Area laws in a many-body localized state and its implications for topological order, J. Stat. Mech.: Theory Exp. 2013, P09005 (2013).

[75] S. A. Parameswaran, A. C. Potter, and R. Vasseur, Eigenstate phase transitions and the emergence of universal dynamics in highly excited states, Annalen der Physik 529, 1600302 (2017).

[76] V. Oganesyan and D. A. Huse, Localization of interacting fermions at high temperature, Phys. Rev. B 75, 155111 (2007).

[77] Y. Y. Atas, E. Bogomolny, O. Giraud, and G. Roux, Distribution of the Ratio of Consecutive Level Spacings in Random Matrix Ensembles, Phys. Rev. Lett. 110, 084101 (2013).

[78] S. F. Edwards and P. W. Anderson, Theory of spin glasses, J. Phys. F: Met. Phys. 5, 965 (1975).

[79] J. A. Kjäll, J. H. Bardarson, and F. Pollmann, Many-Body Localization in a Disordered Quantum Ising Chain, Phys. Rev. Lett. 113, 107204 (2014).

[80] P. Hauke and M. Heyl, Many-body localization and quantum ergodicity in disordered long-range ising models, Phys. Rev. B 92, 134204 (2015).

[81] J. Smith, A. Lee, P. Richerme, B. Neyenhuis, P. W. Hess, P. Hauke, M. Heyl, D. A. Huse, and C. Monroe, Many-body localization in a quantum simulator with programmable random disorder, Nat. Phys. 12, 907 (2016).

[82] M. A. Nielsen and I. L. Chuang, Quantum Computation and Quantum Information: 10th Anniversary Edition (Cambridge University Press, USA, 2011), 10th ed.

[83] N. Gisin, Quantum Measurements and Stochastic Processes, Phys. Rev. Lett. 52, 1657 (1984).

[84] J. Dalibard, Y. Castin, and K. Mølmer, Wave-Function Approach to Dissipative Processes in Quantum Optics, Phys. Rev. Lett. 68, 580 (1992).

[85] T. A. Brun, Continuous measurements, quantum trajectories, and decoherent histories, Phys. Rev. A 61, 042107 (2000).

[86] In practice, a rate of measurement error $p_{\text {meas }}$ increases the number of samples required, $N_{s}$, by a factor of $1 /(1-$ $\left.p_{\text {meas }}\right)^{2}$-i.e., by about $5 \%$ for $p_{\text {meas }}=2.5 \%$.

[87] Google AI Quantum, To appear, (2021). 
[88] R. Roy and F. Harper, Periodic table for floquet topological insulators, Phys. Rev. B 96, 155118 (2017).

[89] M. S. Rudner, N. H. Lindner, E. Berg, and M. Levin, Anomalous Edge States and the Bulk-Edge Correspondence for Periodically Driven Two-Dimensional Systems, Phys. Rev. X 3, 031005 (2013).

[90] H. C. Po, L. Fidkowski, T. Morimoto, A. C. Potter, and A. Vishwanath, Chiral Floquet Phases of Many-Body Localized Bosons, Phys. Rev. X 6, 041070 (2016).

[91] F. Nathan, D. Abanin, E. Berg, N. H. Lindner, and M. S. Rudner, Anomalous floquet insulators, Phys. Rev. B 99, 195133 (2019).

[92] W. Brown and O. Fawzi, Scrambling speed of random quantum circuits, arXiv:1210.6644 (2012).

[93] P. Hosur, X.-L. Qi, D. A. Roberts, and B. Yoshida, Chaos in quantum channels, J. High Energ Phys. 2, 4 (2016).

[94] A. Nahum, S. Vijay, and J. Haah, Operator Spreading in Random Unitary Circuits, Phys. Rev. X 8, 021014 (2018).

[95] C. W. von Keyserlingk, T. Rakovszky, F. Pollmann, and S. L. Sondhi, Operator Hydrodynamics, Otocs, and Entanglement Growth in Systems Without Conservation Laws, Phys. Rev. X 8, 021013 (2018).

[96] H. Gharibyan, M. Hanada, S. H. Shenker, and M. Tezuka, Onset of random matrix behavior in scrambling systems, J. High Energy Phys. 2018, 124 (2018).

[97] V. Khemani, D. A. Huse, and A. Nahum, Velocitydependent lyapunov exponents in many-body quantum, semi-classical, and classical chaos, Phys. Rev. B 98, 144304 (2018).

[98] A. Chan, A. De Luca, and J. T. Chalker, Solution of a Minimal Model for Many-Body Quantum Chaos, Phys. Rev. X 8, 041019 (2018).

[99] B. Bertini, P. Kos, and T. c. v. Prosen, Exact Spectral Form Factor in a Minimal Model of Many-Body Quantum Chaos, Phys. Rev. Lett. 121, 264101 (2018).

[100] A. Nahum, J. Ruhman, S. Vijay, and J. Haah, Quantum Entanglement Growth under Random Unitary Dynamics, Phys. Rev. X 7, 031016 (2017).

[101] T. Rakovszky, F. Pollmann, and C. W. von Keyserlingk, Diffusive Hydrodynamics of Out-Of-Time-Ordered Correlators with Carge Conservation, Phys. Rev. X 8, 031058 (2018).

[102] V. Khemani, A. Vishwanath, and D. A. Huse, Operator Spreading and the Emergence of Dissipative Hydrodynamics under Unitary Evolution with Conservation Laws, Phys. Rev. X 8, 031057 (2018).

[103] A. Pal and D. A. Huse, Many-body localization phase transition, Phys. Rev. B 82, 174411 (2010).

[104] D. J. Luitz, N. Laflorencie, and F. Alet, Many-body localization edge in the random-field heisenberg chain, Phys. Rev. B 91, 081103 (2015).

[105] A. Chandran, C. R. Laumann, and V. Oganesyan, Finite size scaling bounds on many-body localized phase transitions, arXiv:1509.04285 (2015).

[106] V. Khemani, S. P. Lim, D. N. Sheng, and D. A. Huse, Critical Properties of the Many-Body Localization Transition, Phys. Rev. X 7, 021013 (2017).

[107] J. Šuntajs, J. Bonča, T. Prosen, and L. Vidmar, Quantum chaos challenges many-body localization, Phys. Rev. E 102, 062144 (2019).
[108] D. A. Abanin, J. H. Bardarson, G. De Tomasi, S. Gopalakrishnan, V. Khemani, S. A. Parameswaran, F. Pollmann, A. C. Potter, M. Serbyn, and R. Vasseur, Distinguishing localization from chaos: challenges in finite-size systems, Ann. Phys. 427, 168415 (2021).

[109] R. K. Panda, A. Scardicchio, M. Schulz, S. R. Taylor, and M. Žnidarič, Can we study the many-body localisation transition?, EPL (Europhysics Letters) 128, 67003 (2020).

[110] P. Sierant, D. Delande, and J. Zakrzewski, Thouless Time Analysis of Anderson and Many-Body Localization Transitions, Phys. Rev. Lett. 124, 186601 (2020).

[111] M. Schreiber, S. S. Hodgman, P. Bordia, H. P. Lüschen, M. H. Fischer, R. Vosk, E. Altman, U. Schneider, and I. Bloch, Observation of many-body localization of interacting fermions in a quasirandom optical lattice, Science 349, 842 (2015).

[112] S. S. Kondov, W. R. McGehee, W. Xu, and B. DeMarco, Disorder-Induced Localization in a Strongly Correlated Atomic Hubbard gas, Phys. Rev. Lett. 114, 083002 (2015)

[113] J.-y. Choi, S. Hild, J. Zeiher, P. Schauß, A. Rubio-Abadal, T. Yefsah, V. Khemani, D. A. Huse, I. Bloch, and C. Gross, Exploring the many-body localization transition in two dimensions, Science 352, 1547 (2016).

[114] P. Bordia, H. Lüschen, S. Scherg, S. Gopalakrishnan, M. Knap, U. Schneider, and I. Bloch, Probing Slow Relaxation and Many-Body Localization in Two-Dimensional Quasiperiodic Systems, Phys. Rev. X 7, 041047 (2017).

[115] H. P. Lüschen, P. Bordia, S. Scherg, F. Alet, E. Altman, U. Schneider, and I. Bloch, Observation of Slow Dynamics near the Many-Body Localization Transition in One-Dimensional Quasiperiodic Systems, Phys. Rev. Lett. 119, 260401 (2017).

[116] A. Lukin, M. Rispoli, R. Schittko, M. E. Tai, A. M. Kaufman, S. Choi, V. Khemani, J. Léonard, and M. Greiner, Probing entanglement in a many-body-localized system, Science 364, 256 (2019).

[117] M. Rispoli, A. Lukin, R. Schittko, S. Kim, M. E. Tai, J. Léonard, and M. Greiner, Quantum critical behaviour at the many-body localization transition, Nature 573, 385 (2019).

[118] B. Chiaro et al., Direct measurement of non-local interactions in the many-body localized phase, arXiv:1910.06024 (2019).

[119] J. H. Bardarson, F. Pollmann, and J. E. Moore, Unbounded Growth of Entanglement in Models of Many-Body Localization, Phys. Rev. Lett. 109, 017202 (2012).

[120] M. Serbyn, Z. Papić, and D. A. Abanin, Universal Slow Growth of Entanglement in Interacting Strongly Disordered Systems, Phys. Rev. Lett. 110, 260601 (2013).

[121] S. Aaronson, https://www.scottaaronson.com/blog/?p= 4317 (2019), online; accessed: 2020-07-17.

[122] J. Janarek, D. Delande, and J. Zakrzewski, Discrete disorder models for many-body localization, Phys. Rev. B 97, 155133 (2018).

Correction: The Acknowledgment section needed clarification and has been set right. 